\title{
Drafting a cost-effective approach towards a sustainable manufacturing system design
}

\author{
Reda Ahmed O. Nujoom, Ahmed Maher Mohammed \& Qian Wang
}

\begin{abstract}
Decision makers are being increasingly motivated to improve their manufacturing systems in coping efficiently with the objectives of sustainable development and the current manufacturing system designers or researchers face a choice to either incorporate the new regulations of sustainability into the existing systems or leave the field for new players. Consideration of sustainability aspects in developing could potentially reduce the impact of environmental wasters. Design of a sustainable manufacturing systems (SMS) can be partially achieved through the implementation of lean methods to reduce manufacturing wastes and operational costs, while improving system productivity. On the other hand, such methods of leanness does not encounter environmental wastes particularly, energy usage and carbon dioxide $\left(\mathrm{CO}_{2}\right)$ emissions of a lean manufacturing system. This work overcomes these shortcomings in developing a SMS towards the minimization of total cost, energy consumption and environmental impact, in particular, of the $\mathrm{CO}_{2}$ emissions. The design problem was modelled as a multi-objective programming model under economic and ecological constraints. The developed model is also associated with the number of machines required for operating processes along with the quantity of flow of material for processing the products in a manufacturing system. An integrated decision-making trial and evaluation laboratory (DEMATEL)- $\varepsilon^{-}$ constraint approach and the goal programming approach were used to derive two sets of non-inferior solutions. Finally, a real case study was used for examining the applicability of the developed SMS model.
\end{abstract}

Keywords: Sustainability; Multi-objective optimization; DEMATEL; $\mathrm{CO}_{2}$ emissions; lean manufacturing. 


\section{INTRODUCTION}

To design a SMS, decision makers need to incorporate the environmentalism aspect in addition to conventional aspects in improving system efficiency and productivity (Lind et al., 2008). The conventional manufacturing system configuration is normally associated with parameters of operating capacities, products flow, material-handling and production methods, operations and shop-floor layouts. However, the growing interest in environmentalism has encouraged decision makers to incorporate environmental aspects which represents an additional challenge for designers to obtain a cost-effective and environmental-friendly method (Paju et al., 2010). The terms of SMS was used to refer to a compromised between the environmental and economic aspects (Taghdisian et al., 2014). The manufacturing sustainability can be presented as production of manufactural goods while curtailing detrimental environmental effects relating to energy usage or depletion of natural resources. Thus, the minimization of environmental impact ought to be presented as a separate objective along with other traditional objectives such as minimizing total cost and maximizing service level and system efficiency, which form a multi-objective optimization problem.

Development of a SMS may be partially achieved by applying lean methods as a trend in modern manufacturing companies to enhance system performance adding no extra investment. Leanness can be defined as "a systematic approach to eliminate non-value added wastes in various forms and it enables continuous improvement" (Dombrowski et al., 2014). These wastes are waiting for parts to arrive, overproduction, unnecessary movement of materials, unnecessary inventory, excess motion, the waste in processing and the waste of rework (Wang et al., 2009). However, leanness does not encounter energy usage and $\mathrm{CO}_{2}$ emissions in presenting a lean manufacturing system. (Wang et al., 2009). Subsequently, it is a paramount to develop a sustainable lean manufacturing system incorporating the economic and ecological constraints as industrial factories consume energy and subsequently produce $\mathrm{CO}_{2}$ emissions.

This paper contributes to the literature through in formulating a multi-objective model to achieve the optimal configuration of the proposed SMS design seeking a trade-off among the three objectives. The aim of objectives was to minimize the total manufacturing system cost, energy consumption due to operating machines, airconditioning units and illumination bulbs in manufacturing processes and the $\mathrm{CO}_{2}$ emissions released from 
those equipment and from the transportation vehicles. The formulated model was coded using $\mathrm{LINGO}^{11}$ in which non-inferior solutions were obtained using an integrated DEMATEL- $\varepsilon$-constraint and goal programming approaches. Unlike other researches, the most important objective used to apply the $\varepsilon$-constraint approach is determined by using the DEMATEL algorithm which is to our knowledge a new contribution.

The rest of this article is organised as follows: Section 2 presents the literature review on sustainable manufacturing and multi-objective optimization. Section 3 describes the development of the multi-objective model. Section 4 illustrates the proposed optimization methodology. Section 5 shows an application of the developed multi-objective model and the proposed optimization methodology in a real case study and Section 6 concludes and suggests avenues for future work.

\section{Literature review}

There are a few studies in considering environmental aspects related to design of manufacturing systems or SMS. Heilala et al., (2008) mentioned that designers of manufacturing system have to rely not just on those methods traditionally used to enhance how efficient and productive the system will be, but need to also analyze impacts that the designed system will have on the environment and surroundings. Wang et al., (2008) developed an approach so-called process integration employed for examining the environmental impact for a steel company. Branham et al., (2008) measured various categories of energy consumption in manufacturing system using the quantitative thermodynamic analysis. Guillen-Gosalbez and Grossmann (2009) formulated a scenario-based two objectives model employed for designing a sustainable chemical supply chain towards a minimum environmental impact.

A SMS design problem can be modelled as a multi objectives programming model towards the optimization of objectives (e.g., profits and service level). Sahar et al., (2014) formulated a multiple objectives model used for minimizing $\mathrm{CO}_{2}$ emissions of transportation and the total cost for product distribution of a dairy supply chain. Vahdani et al., (2012) developed a two objectives optimization model in assisting the design of a supply chain by minimizing costs of facilities and transportation as objectives. Abdallah et al., (2010) formulated a supply chain network in terms of a multiple objectives programming model aimed at minimizing environmental impact and costs. Kannan et al., (2012) developed an integrated, multi echelon, multi period, 
multi-product optimization model used for optimizing the distribution and inventory level of a supply chain network. Zhang et al., (2015) proposed a dynamical optimization method for shop-floor material handling based on real-time and multi-source manufacturing data. It integrates three important features including a new allocation strategy for move tasks, intelligent trolleys with the capability of active sensing and self-decision and the combination optimization method of move tasks to reduce the transport cost and energy. A study by Wang et al., (2011) included the development of a multiple objectives model for determining a trade-off decision comparing the overall costs incurred versus the total $\mathrm{CO}_{2}$ emitted from facilities within the supply chain. Also, as developed by Jamshidi et al., (2012), a multiple objectives model was formulated considering the annual cost minimization and the effects of nitrogen dioxide, carbon monoxide and volatile organic particles caused by production at facilities and transportation in the supply chain. Niknamfar (2015) formulated a multiple objectives model optimized by using two genetic algorithms for addressing a production-distribution planning problem of a three-level supply chain. Bortolini et al., (2016) formulated a multi-objective programming model to minimize operating cost, carbon footprint and delivery time in a fresh food distribution network.

To summarize, previous studies show the importance of incorporating sustainability when evaluating the performance of manufacturing system. However, there is a gap in this body of knowledge in combining lean manufacturing and environmental waste to create a balance under the economic and ecological constraints. To the best of authors' knowledge there is no study yet developed a multi objective optimization approach combining lean manufacturing and environmental impact to deal with the environmental problems as this problem still at an infant stage.

\section{Developing the multi-objective model}

Figure 1 shows the configuration of a SMS design in which three facilities were considered, these are supplier $s$, factory $f$ and warehouse $w$. The facility (i.e., suppliers and factories) may consist of operation machines, along with air-conditioning units, bulbs and various related equipment including the compressors that supplies some of these machines with compressed air. Between facilities, there are transportation vehicles to be used. In order to achieve a SMS, energy consumption of all manufacturing equipment as well as total 
amount of $\mathrm{CO}_{2}$ emissions need to be determined, along with total manufacturing system cost. The design problem was modelled as a multi-objective model to:

1. Obtain a compromised solution among the three objectives (i.e., minimum total manufacturing system cost (equation 1), energy consumption (equation 2), and $\mathrm{CO}_{2}$ emissions (equation 3 ) as described below

2. Determine the optimal numbers of operation machines

3. Determine the optimal quantity of materials flows in the manufacturing system

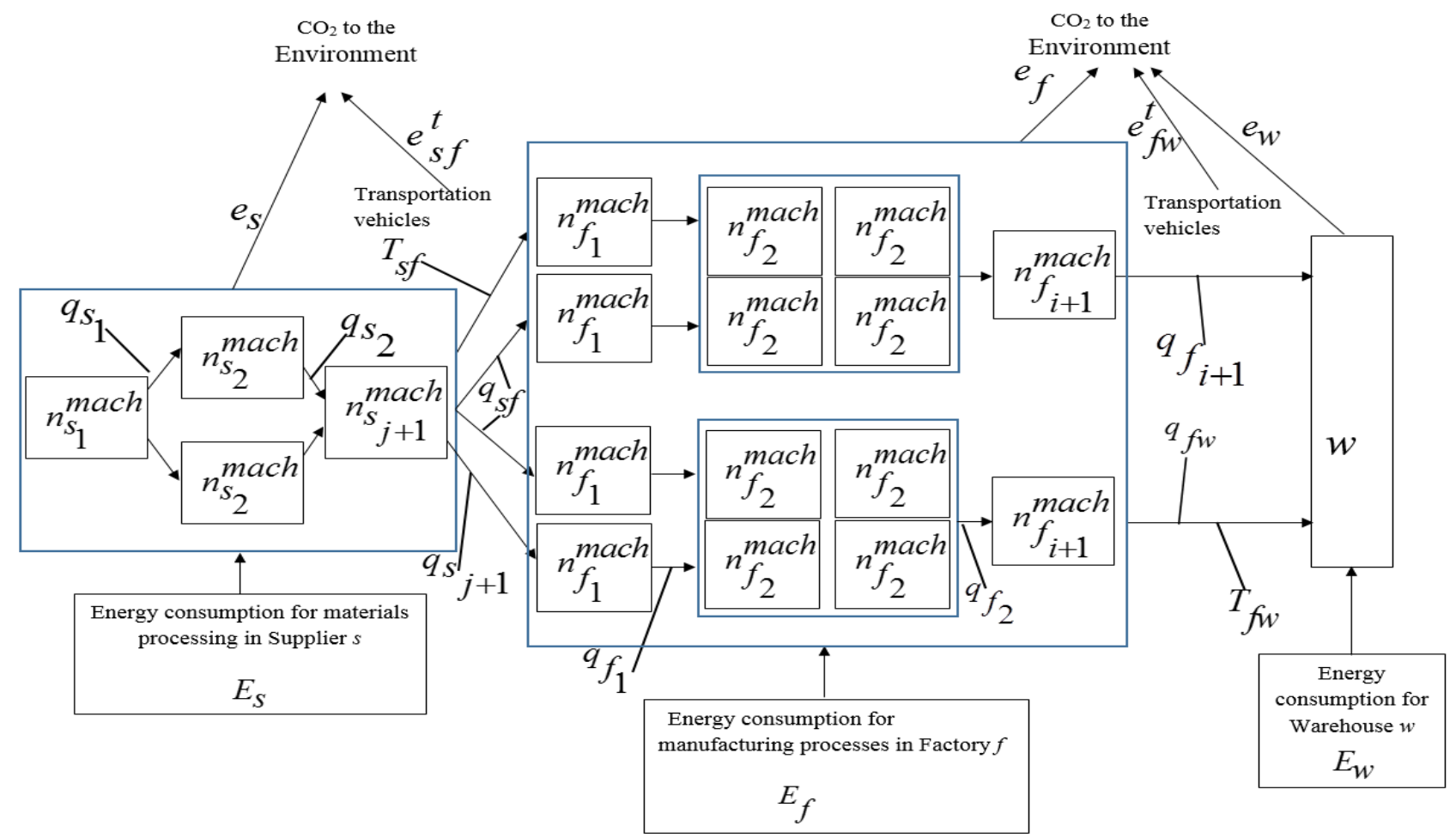

Fig.1. Configuration of the sustainable manufacturing system 
Sets:

$S$

$f$

w

$\Pi_{s}$ and $\Pi_{f}$

\section{Parameters}

$C_{l}^{e s}$

$C_{s}^{\text {mach }}$ and $C_{f}^{\text {mach }}$

$C_{l}^{\text {cond }}$

$C_{l}^{b u l p}$

$C_{S}^{r}$

$C_{s f}^{r}$

$C_{f}^{m p}$

$C_{f w}^{m p}$

$C_{w}^{I}$ set of supplier $s(1 \ldots s \ldots S)$

set of factory $f(1 \ldots f \ldots F)$

set of warehouse $w(1 \ldots s \ldots W)$

number of manufacturing processes involved in supplier $s$

and factory $f$ respectively.

cost required (GBP) for establishing facility $l$, where $l \in\{s, f, w\}$

cost of machines (GBP) involved in processes $j$ in facility $s$ and $i$ in facility $f$ respectively.

cost of an air-conditioning units (GBP) in facility $l$

cost of a lighting bulbs (GBP) in facility $l$

unit raw materials cost (GBP) at supplier $s$

total raw materials cost (GBP) from supplier $s$ to factory $f$

unit manufacturing product cost (GBP) at factory $f$

total manufacturing product cost (GBP) from factory $f$ to warehouse $w$

unit inventory cost (GBP) per product at warehouse $w$ 


$$
\begin{gathered}
C_{f w}^{I} \\
C_{l}^{t}
\end{gathered}
$$$$
C_{s f}^{t} \text { and } C_{f w}^{t}
$$$$
T_{s f} \text { and } T_{f w}
$$

$\mathrm{Ca}$

$$
D_{f} \text { and } D_{w}
$$$$
E_{S}, E_{f} \text { and } E_{w}
$$$$
E_{s_{j}}^{\text {mach }} \text { and } E_{f_{i}}^{\text {mach }}
$$$$
E_{S_{j}}^{\text {comp }} \text { and } E_{f_{i}}^{c o m p}
$$

$$
E_{s_{j}}^{c o n d} \text { and } E_{f_{i}}^{c o n d}
$$

total inventory cost (GBP) from factory $f$ to warehouse $w$

unit transportation cost (GBP) of transportation raw materials and product per mile between facilities $l$

total transportation cost (GBP) of raw material and products per mile from supplier $s$ to factory $f$ and from factory $f$ to warehouse $w$ respectively

transportation distance (miles) from supplier $s$ to factory $f$ and from factory $f$ to warehouse $w$

operations capacity $(\mathrm{kg})$ of facility $l$

demand (kg) of factory $f$ and warehouse $w$ respectively

energy consumption (kWh) for supplier $s$, for factory $f$ and for warehouse $w$ respectively

energy consumption $(\mathrm{kWh})$ for a machine in processes $j$ in supplier $s$ and $i$ in factory $f$ respectively, where $j \in\left\{1,2, \ldots ., \Pi_{s}\right\}$ and $i \in\left\{1,2, \ldots ., \Pi_{f}\right\}$ energy consumption $(\mathrm{kWh})$ of compressed air needed for a machine in processes $j$ in supplier $s$ and $i$ in factory $f$ respectively

energy consumption $(\mathrm{kWh})$ for the air-conditioning units in processes $j$ in supplier $s$ and $i$ in factory $f$ respectively 


$$
E_{s_{j}}^{b u l b} \text { and } E_{f_{i}}^{b u l b}
$$$$
E_{w}^{c o n d} \text { and } E_{w}^{b u l b}
$$$$
N_{s_{j}}^{\text {mach }} \text { and } N_{f_{i}}^{\text {mach }}
$$$$
\Re_{s_{j}} \text { and } \Re_{f_{i}}
$$

$$
\tau_{s_{j}} \text { and } \tau_{f_{i}}
$$$$
\mu_{s_{j}} \text { and } \mu_{f_{i}}
$$$$
N_{s_{j}}^{c o m p} \text { and } N_{f_{i}}^{c o m p}
$$$$
N_{s_{j}}^{\text {cond }} \text { and } N_{f_{i}}^{\text {cond }}
$$$$
N_{s_{j}}^{b u l b} \text { and } N_{f_{i}}^{\text {bulb }}
$$$$
\wp_{s}, \wp_{f} \text { and } \wp_{w}
$$$$
\Psi_{s_{j}} \text { and } \Psi_{f_{i}}
$$

energy consumption $(\mathrm{kWh})$ for the lighting bulbs in processes $j$ in supplier $s$ and $i$ in factory $f$ respectively

energy consumption $(\mathrm{kWh})$ for the air-conditioning units and lighting bulbs in warehouse $w$ respectively

installed power $(\mathrm{kw})$ for a machine in processes $j$ in supplier $s$ and $i$ in factory $f$ respectively

manufacturing rate $(\mathrm{kg} / \mathrm{h})$ for a machine in processes $j$ in supplier $s$ and $i$ in factory $f$ respectively

operating time (hr) for a machine in processes $j$ in supplier $s$ and $i$ in factory $f$ respectively

efficiency (\%) for a machine in processes $j$ in supplier $s$ and $i$ in factory $f$ respectively

installed power (kw) for a compressor in supplier $s$ and factory $f$ respectively

installed power $(\mathrm{kw})$ for an air-conditioning unit in processes $j$ in supplier $s$ and $i$ in factory $f$ respectively

installed power $(\mathrm{kw})$ for a lighting bulb in processes $j$ in supplier $s$ and $i$ in factory $f$ respectively

mass production $(\mathrm{kg} / \mathrm{month})$ from supplier $s$, from factory $f$ and stored in warehouse $w$ respectively

total waste ratio (\%) for a machine involved in processes $j$ in supplier $s$ and $i$ in factory $f$ respectively 
$v_{s_{j}}^{c o m p}$ and $v_{f_{i}}^{c o m p}$

$\rho_{s}^{c o m p}$ and $\rho_{f}^{c o m p}$

$\Phi_{s_{j}}^{\text {cond }}$ and $\Phi_{f_{i}}^{\text {cond }}$

$\varphi_{s_{j}}^{b u l b}$ and $\varphi_{f_{i}}^{b u l b}$

$\Gamma_{w}^{\text {cond }}$

$\lambda_{w}^{b u l b}$

$e_{s_{j}}^{m a c h}$ and $e_{f_{i}}^{m a c h}$

$e_{s_{j}}^{c o m p}$ and $e_{f_{i}}^{c o m p}$

$e_{s_{j}}^{c o n d}$ and $e_{f_{i}}^{\text {cond }}$

$e_{s_{j}}^{b u l b}$ and $e_{f_{i}}^{b u l b}$ compressed air $\left(\mathrm{m}^{3} / \mathrm{h}\right)$ used for a machine in processes $j$ in supplier $s$ and $i$ in factory $f$ respectively

capacity of a compressor $\left(\mathrm{m}^{3} / \mathrm{h}\right)$ in supplier $s$ and factory $f$ respectively

covering rate per air-conditioning unit (unit) that serves machines in processes $j$ in supplier $s$ and $i$ in factory $f$ respectively

covering rate of lighting bulbs (unit) per one machine processes $j$ in supplier and $i$ in factory $f$ respectively

covering rate per air-conditioning unit $(\mathrm{kg})$ that services quantity of products in warehouse $w$

covering rate per lighting bulb $(\mathrm{kg})$ that serves quantity of products in warehouse $w$

amount of $\mathrm{CO}_{2}$ emissions $(\mathrm{kg})$ released from the machines in processes $j$ of supplier $s$ and $i$ of factory $f$ respectively

amount of $\mathrm{CO}_{2}$ emissions $(\mathrm{kg})$ released from a compressor system in processes $j$ of supplier $s$ and $i$ of factory $f$ respectively

amount of $\mathrm{CO}_{2}$ emissions $(\mathrm{kg})$ released from the air-conditioning units in processes $j$ of supplier $s$ and $i$ of factory $f$ respectively

amount of $\mathrm{CO}_{2}$ emissions $(\mathrm{kg})$ released from the lighting bulbs in processes $j$ of supplier $s$ and $i$ of factory $f$ respectively 
$e_{w}^{c o n d}$ and $e_{w}^{b u l b}$

$e_{s f}^{t}$ and $e_{f w}^{t}$

V

$\omega_{s_{j}}, \omega_{f_{i}}$ and $\omega_{w}$

$\omega_{s f}^{t}$ and $\omega_{f w}^{t}$

\section{Decision variables}

$q_{s_{j}}^{r}$ and $q_{f_{i}}^{r}$

$q_{s(j+1)}^{r}$ and $q_{f_{(i+1)}}^{r}$

$q_{s f}^{r}$ and $q_{f w}^{m p}$

$n_{s_{j}}^{\text {mach }}$ and $n_{f_{i}}^{\text {mach }}$

$n_{s_{j}}^{\text {cond }}, n_{f_{i}}^{\text {cond }}$ and $n_{w}^{\text {cond }}$ amount of $\mathrm{CO}_{2}$ emissions $(\mathrm{kg})$ released from air-conditioning units and the lighting bulbs involved in warehouse $w$ respectively

amount of $\mathrm{CO}_{2}$ emissions $(\mathrm{kg})$ released for transportation from supplier $s$ to factory $f$ and from factory $f$ to warehouse $w$ respectively

capacity (units) per vehicle

$\mathrm{CO}_{2}$ emission factor $(\mathrm{kg} / \mathrm{kWh})$ in supplier $s$, in factory $f$ and warehouse $w$ respectively

$\mathrm{CO}_{2}$ emission factor $(\mathrm{kg} / \mathrm{mile})$ released from raw material transportation from supplier $s$ to

factory $f$ and from products transportation from factory $f$ to warehouse $w$ respectively

mass of material $(\mathrm{kg})$ involved in processes $j$ in supplier $s$ and $i$ in factory $f$ respectively where, $j \in\left\{1,2, \ldots ., \Pi_{s}\right\}$ and $i \in\left\{1,2, \ldots ., \Pi_{f}\right\}$

mass of material $(\mathrm{kg})$ transferred from the machines in processes $j$ in supplier $s$ and $i$ in factory $f$ respectively

mass of material $(\mathrm{kg})$ transported from supplier $s$ to factory $f$ and products transported from factory $f$ to warehouse w

number of machines (unit) in processes $j$ in supplier $s$ and $i$ in factory $f$ respectively

number of air-conditioning units (unit) in processes $j$ in supplier $s$ and $i$ in factory $f$ and in warehouse $w$ respectively 
$n_{s_{j}}^{\text {bulb }}, n_{f_{i}}^{\text {bulb }}$ and $n_{w}^{\text {bulb }}$ number of lighting bulbs (unit) in processes $j$ in supplier $s$ and $i$ in factory $f$ and in warehouse $w$ respectively

\section{Minimization of total $\operatorname{cost} Z_{1}$}

$\operatorname{Min} Z_{1}=C_{s}^{e s}+C_{f}^{e s}+C_{w}^{e s}+C_{s}^{\text {mach }}$

$+C_{f}^{\text {mach }}+C_{s}^{\text {cond }}+C_{f}^{\text {cond }}+C_{w}^{\text {cond }}+C_{s}^{\text {bulp }}$

$+C_{f}^{b u l p}+C_{w}^{b u l p}+C_{s f}^{r}+C_{f w}^{m p}+C_{s f}^{t}+C_{f w}^{t}+C_{f w}^{I}$

Where, cost required for establishing supplier $s$, factory $f$ and warehouse $w\left(C_{S}^{e s}, C_{f}^{e s}\right.$ and $\left.C_{w}^{e s}\right)$ is given respectively as follows:

$C_{S}^{e s}=C_{S}^{\text {land }}+C_{S}^{\text {building }}$

$+C_{S}^{\text {equipment }}+C_{S}^{\text {services }}+C_{S}^{\text {saleries }}$

$C_{f}^{e s}=C_{f}^{\text {land }}+C_{f}^{\text {building }}$

$+C_{f}^{\text {equipment }}+C_{f}^{\text {services }}+C_{f}^{\text {saleries }}$

$C_{w}^{e s}=C_{w}^{\text {land }}+C_{w}^{\text {building }}$

$+C_{w}^{\text {equipment }}+C_{w}^{\text {services }}+C_{w}^{\text {saleries }}$

Cost of the machines $C_{s}^{\text {mach }}$ and $C_{f}^{\text {mach }}$ involved in process $j$ at supplier $s$ and in process $i$ at factory $f$ is given respectively as follows:

$$
\begin{aligned}
& C_{s}^{\text {mach }}=\sum_{j=1}^{\prod_{s}}\left(C_{s_{j}}^{\text {mach }} n_{s_{j}}^{\text {machin }}\right) \\
& C_{f}^{\text {mach }}=\sum_{i=1}^{\Pi_{f}}\left(C_{f_{i}}^{\text {mach }} n_{f_{i}}^{\text {machin }}\right)
\end{aligned}
$$


Cost of an air-conditioning unit $C_{S}^{\text {cond }}, C_{f}^{c o n d}$ and $C_{w}^{c o n d}$ involved in process $j$ at supplier $s$, in process $i$ at factory $f$ and at warehouse $w$ is determined respectively as follows:

$$
\begin{aligned}
& C_{s}^{\text {cond }}=\sum_{j=1}^{\prod_{S}}\left(C_{s_{j}}^{\text {cond }} n_{s_{j}}^{\text {cond }}\right) \\
& C_{f}^{\text {cond }}=\sum_{j=1}^{\prod_{f}}\left(C_{f_{i}}^{\text {cond }} n_{f_{i}}^{\text {cond }}\right) \\
& C_{w}^{\text {cond }}=\sum_{w=1}^{W}\left(C_{w}^{\text {cond }} n_{w}^{\text {cond }}\right)
\end{aligned}
$$

Cost of a lighting bulb $C_{S}^{b u l p}, C_{f}^{b u l p}$ and $C_{w}^{b u l p}$ in process $j$ at supplier $s$, in process $i$ at factory $f$ and at warehouse $w$ is determined respectively as follows:

$$
\begin{aligned}
& C_{s}^{\text {bulp }}=\sum_{j=1}^{\Pi_{S}}\left(C_{s_{j}}^{\text {bulp }} n_{s_{j}}^{\text {bulb }}\right) \\
& C_{f}^{\text {bulp }}=\sum_{i=1}^{\prod_{f}}\left(C_{f_{i}}^{\text {bulp }} n_{f_{i}}^{\text {bulb }}\right) \\
& C_{w}^{\text {bulp }}=\sum_{w=1}^{W}\left(C_{w}^{\text {bulp }} n_{w}^{\text {bulb }}\right)
\end{aligned}
$$

Total cost of raw materials at supplier $s C_{s f}^{r}$ is calculated as below:

$$
C_{s f}^{r}=\sum_{S=1}^{S} \sum_{f=1}^{F} C_{S}^{r} q_{s f}^{r}
$$

Total cost of manufacturing products at factory $f C_{f w}^{m p}$ is determined as follows:

$$
C_{f w}^{m p}=\sum_{f=1}^{F} \sum_{w=1}^{W} C_{f}^{m p} q_{f w}^{m p}
$$


Total cost of transportation of raw materials per mile between $s$ and $f C_{s f}^{t}$ is determined as

follows:

$C_{s f}^{t}=\sum_{s=1}^{S} \sum_{f=1}^{F} C_{s f}^{t} \frac{q_{s f}^{r}}{V} T_{s f}$

Total cost of transportation of products per mile between $f$ and $w C_{f w}^{t}$ is determined as follows:

$C_{f w}^{t}=\sum_{f=1}^{F} \sum_{w=1}^{W} C_{f w}^{t} \frac{q_{f w}^{m p}}{V} T_{f w}$

Total cost of inventory $C_{f w}^{I}$ at warehouse $w$ is determined as follows:

$C_{f w}^{I}=\sum_{f=1}^{F} \sum_{w=1}^{W} C_{w}^{I} q_{f w}^{m p}$

Hence, equation 1 can be expressed as follows:

Min $\mathrm{Z}_{1}=C_{S}^{\text {land }}+C_{S}^{\text {building }}+C_{S}^{\text {equipment }}+C_{S}^{\text {services }}+C_{S}^{\text {saleries }}$

$+C_{f}^{\text {land }}+C_{f}^{\text {building }}+C_{f}^{\text {equipment }}+C_{f}^{\text {services }}+C_{f}^{\text {saleries }}+C_{w}^{\text {land }}$

$+C_{w}^{\text {building }}+C_{w}^{\text {equipment }}+C_{w}^{\text {services }}+C_{w}^{\text {saleries }}+\sum_{j=1}^{\Pi_{s}}\left(C_{s_{j}}^{\text {mach }} n_{s_{j}}^{\text {mach }}\right)$

$+\sum_{i=1}^{\Pi_{f}}\left(C_{f_{i}}^{\text {mach }} n_{f_{i}}^{\text {mach }}\right)+\sum_{j=1}^{\Pi_{s}}\left(C_{s_{j}}^{\text {cond }} n_{s_{j}}^{\text {cond }}\right)+\sum_{j=1}^{\Pi_{f}}\left(C_{f_{i}}^{\text {cond }} n_{f_{i}}^{\text {cond }}\right)$

$+\sum_{w=1}^{W}\left(C_{w}^{\text {cond }} n_{w}^{\text {cond }}\right)+\sum_{j=1}^{\Pi_{s}}\left(C_{s_{j}}^{\text {bulp }} n_{s_{j}}^{\text {bulb }}\right)+\sum_{i=1}^{\Pi_{f}}\left(C_{f_{i}}^{\text {bulp }} n_{f_{i}}^{\text {bulb }}\right)$

$+\sum_{w=1}^{W}\left(C_{w}^{b u l p} n_{w}^{b u l b}\right)+\sum_{s=1}^{S} \sum_{f=1}^{F} C_{s}^{r} q_{s f}^{r}+\sum_{f=1}^{F} \sum_{w=1}^{W} C_{f}^{m p} q_{f w}^{m p}$

$+\sum_{s=1}^{S} \sum_{f=1}^{F} C_{s f}^{t} \frac{q_{s f}^{r}}{V} T_{s f}+\sum_{f=1}^{F} \sum_{w=1}^{W} C_{f w}^{t} \frac{q_{f w}^{m p}}{V} T_{f w}+\sum_{f=1}^{F} \sum_{w=1}^{W} C_{w}^{I} q_{f w}^{m p}$

\section{Minimization of energy consumption $Z_{2}$}

$\operatorname{Min} Z_{2}=\sum_{j=1}^{\prod_{s}}\left(E_{s_{j}}^{\text {mach }}+E_{s_{j}}^{\text {cond }}+E_{s_{j}}^{\text {bulb }}+E_{s_{j}}^{\text {comp }}\right)$

$+\sum_{i=1}^{\Pi_{f}}\left(E_{f_{i}}^{\text {mach }}+E_{f_{i}}^{\text {cond }}+E_{f_{i}}^{\text {bulb }}+E_{f_{i}}^{\text {comp }}\right)+E_{w}^{\text {cond }}+E_{w}^{\text {bulb }}$ 
Where,

Energy consumption $E_{S_{j}}^{\text {mach }}, E_{S_{j}}^{\text {cond }}$ and $E_{S_{j}}^{b u l b}$ for machines, air-conditioning units and

lighting bulbs in process $j$ at supplier $s$ is given respectively by:

$$
\begin{aligned}
& E_{s_{j}}^{\text {mach }}=\sum_{j=1}^{\Pi_{s}}\left(\frac{q_{s_{j}}^{r}}{\Re_{s_{j}} \mu_{s_{j}}} N_{s_{j}}^{\text {mach }} n_{s_{j}}^{\text {mach }}\right) \\
& E_{S_{j}}^{\text {cond }}=\sum_{j=1}^{\Pi_{S}}\left(N_{s_{j}}^{\text {cond }} n_{s_{j}}^{\text {cond }} \frac{q_{S_{(j+1)}^{r}}^{r}}{\wp_{S}}\right) \\
& E_{s_{j}}^{b u l b}=\sum_{j=1}^{\Pi_{S}}\left(N_{s_{j}}^{b u l b} n_{s_{j}}^{b u l b} \frac{q_{s_{(j+1)}^{r}}^{r}}{\wp_{s}}\right)
\end{aligned}
$$

Energy consumption for machines in process $j$ at supplier $s$, is calculated by:

$$
E_{s_{j}}^{\text {comp }}=\sum_{j=1}^{\Pi_{s}}\left(\frac{q_{s_{j}}^{r}}{\Re_{s_{j}} \mu_{s_{j}}} \frac{N_{s_{j}}^{\text {comp }}}{\rho_{s_{j}}^{\text {comp }}} v_{s_{j}}^{\text {comp }} n_{s_{j}}^{\text {mach }}\right)
$$

Energy consumption for machines, air-conditioning units and lighting bulbs in process $i$ at factory $f$ is determined respectively by:

$$
\begin{aligned}
& E_{f_{i}}^{\text {mach }}=\sum_{i=1}^{\prod_{f}}\left(\frac{q_{f_{i}}^{r}}{\Re_{f_{i}} \mu_{f_{i}}} N_{f_{i}}^{\text {mach }} n_{f i}^{\text {mach }}\right) \\
& E_{f_{i}^{\text {cond }}}^{\prod_{f}}=\sum_{i=1}^{\Pi_{f}}\left(N_{f_{i}}^{\text {cond }} n_{f_{i}}^{\text {cond }} \frac{q_{f_{(i+1)}}^{r}}{\wp f}\right) \\
& E_{f_{i}}^{\text {bulb }}=\sum_{i=1}^{\Pi_{f}}\left(N_{f_{i}}^{\text {bulb } n_{f_{i}}^{\text {bulb }}} \frac{q_{f_{(i+1)}^{r}}^{\wp_{f}}}{{ }^{\prime}}\right)
\end{aligned}
$$


Energy consumption for machines in process $i$ at factory $f$ is determined as follows:

$E_{f_{i}}^{\text {comp }}=\sum_{i=1}^{\Pi_{f}}\left(\frac{q_{f_{i}}^{r}}{\Re_{f i}{ }_{f i}} \frac{N_{f_{i}}^{\text {comp }}}{\rho_{f_{i}}^{\text {comp }}} v_{f_{i}}^{\text {comp }} n_{f_{i}}^{\text {mach }}\right)$

Energy consumption for air-conditioning units and lighting bulbs at warehouse $w$ is determined as follows:

$E_{w}^{c o n d}=\sum_{w=1}^{W}\left(N_{w}^{c o n d} n_{w}^{c o n d} \frac{q_{f w}^{m p}}{\wp_{w}}\right)$

$E_{w}^{b u l b}=\sum_{w=1}^{W}\left(N_{w}^{b u l b} \times n_{w}^{b u l b} \times \frac{q_{f w}^{m p}}{\wp_{w}}\right)$

Hence, equation 18 is given as follows:

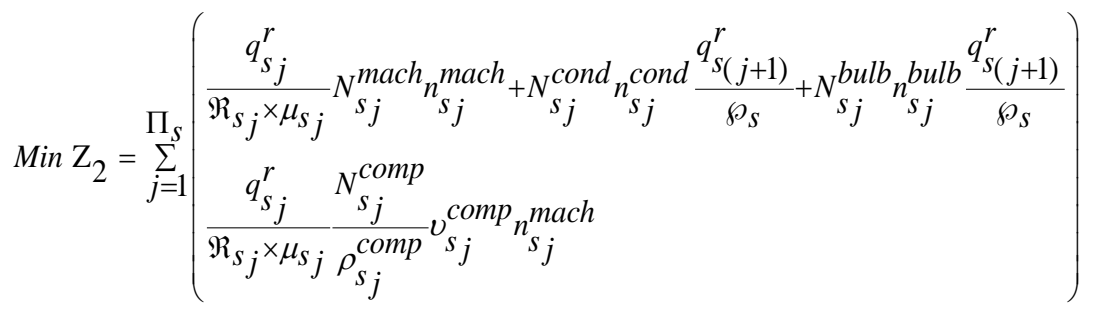

$$
\begin{aligned}
& +\sum_{i=1}\left(\begin{array}{l}
\frac{q_{f_{i}}^{r}}{\Re_{f_{i}} \times \mu_{f_{i}}} N_{f_{i}}^{\text {mach }} n_{f_{i}}^{\text {mach }}+N_{f_{i}}^{\text {cond }} n_{f_{i}}^{\text {cond }} \frac{q_{f_{(i+1)}}^{r}}{\wp f}+N_{f_{i}}^{b u l b} n_{f_{i}}^{\text {bulb }} \frac{q_{f_{(i+1)}}^{r}}{\wp f} \\
+\frac{q_{f_{i}}^{r}}{\Re_{f_{i}} \times \mu f_{i}} \frac{N_{f_{i}}^{c o m p}}{\rho_{f_{i}}^{\text {comp }}} v_{f_{i}}^{\text {comp }} n_{f_{i}}^{\text {mach }}
\end{array}\right) \\
& +\sum_{w=1}^{W}\left(N_{w}^{\text {cond }} n_{w}^{\text {cond }} \frac{q_{w}^{m p}}{\wp_{w}}+N_{w}^{b u l b} n_{w}^{b u l b} \frac{q_{f w}^{m p}}{\wp_{w}}\right)
\end{aligned}
$$

\section{Minimization of $\mathrm{CO}_{2}$ emissions $\mathrm{Z}_{3}$}

$\operatorname{Min} \mathrm{Z}_{3}=\sum_{j=1}^{\Pi_{s}}\left[e_{s_{j}}^{\text {mach }}+e_{s_{j}}^{\text {cond }}+e_{s_{j}}^{\text {bulb }}+e_{s_{j}}^{\text {comp }}\right]+e_{s f}^{t}+e_{f w}^{t}$

$\left.+\sum_{i=1}^{\Pi} f e_{f_{i}}^{\text {mach }}+e_{f_{i}}^{\text {cond }}+e_{f_{i}}^{\text {bulb }}+e_{f_{i}}^{\text {comp }}\right]+e_{w}$ 
Where, amount of $\mathrm{CO}_{2}$ emissions $e_{s_{j}}^{\text {mach }}, e_{s_{j}}^{\text {cond }}$ and $e_{s_{j}}^{\text {bulb }}$ due to operating of machines, airconditioning units and lighting bulbs in process $j$ at supplier $s$ is respectively determined as follows:

$$
\begin{aligned}
& e_{s_{j}}^{\text {mach }}=\sum_{j=1}^{\Pi_{S}}\left(\omega_{s_{j}} \frac{q_{s_{j}}^{r}}{\Re_{s_{j}} \mu_{s_{j}}} N_{s_{j}}^{\text {mach }} n_{s_{j}}^{\text {mach }}\right) \\
& e_{s_{j}}^{\text {cond }}=\sum_{j=1}^{\Pi_{s}}\left(0.689 N_{s_{j}}^{\text {cond }} n_{s_{j}} \text { cond } \frac{q_{s_{(j+1)}}^{r}}{\wp_{s}}\right) \\
& e_{s_{j}}^{b u l b}=\sum_{j=1}^{\Pi_{S}}\left(0.689 N_{s_{j}}^{b u l b} n_{s_{j}}^{b u l b} \frac{q_{s_{(j+1)}}^{r}}{\wp_{s}}\right)
\end{aligned}
$$

Amount of $\mathrm{CO}_{2}$ emissions in process $j$ of supplier $s$ is determined as follows:

$$
e_{s_{j}}^{c o m p}=\sum_{j=1}^{\prod_{S}}\left(0.689 \frac{q_{s_{j}}^{r}}{\mathfrak{R}_{s_{j}} \mu_{s_{j}}} \frac{N_{s_{j}}^{c o m p}}{\rho_{s_{j}}^{c o m p}} v_{s_{j}}^{\text {comp }} n_{s_{j}}^{\text {mach }}\right) \text { where } 0.689 \text { is the emission factor for the }
$$

electricity

Amount of $\mathrm{CO}_{2}$ emissions due to transporting raw material from supplier $s$ to factory $f$ and products from factory $f$ to warehouse $w$ is respectively determined as follows:

$$
\begin{aligned}
& e_{s f}^{t}=\sum_{s=1}^{S} \sum_{f=1}^{F}\left(\omega_{s f}^{t} \frac{q_{s f}^{r}}{V} T_{s f}\right) \\
& e_{f w}^{t}=\sum_{f=1}^{F} \sum_{w=1}^{W}\left(\omega_{f w}^{t} \frac{q_{f w}^{m p}}{V} T_{f w}\right)
\end{aligned}
$$


Amount of $\mathrm{CO}_{2}$ emissions due to operating of machines, air-conditioning units and lighting bulbs in process $i$ at factory $f$ is given respectively by:

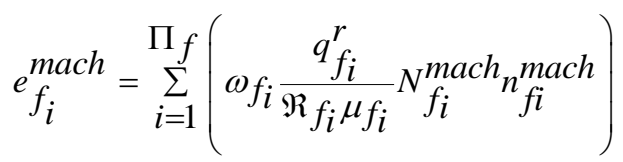

$$
\begin{aligned}
& e_{f_{i}}^{\text {cond }}=\sum_{i=1}^{\Pi_{f}}\left(0.689 N_{f_{i}}^{\text {cond }} n_{f_{i}}^{\text {cond }} \frac{q_{f_{(i+1)}^{r}}^{r}}{\wp f}\right) \\
& e_{f_{i}}^{b u l b}=\sum_{i=1}^{\Pi_{f}}\left(0.689 N_{f_{i}}^{b u l b} n_{f_{i}}^{b u l b} \frac{q_{f_{(i+1)}}^{r}}{\wp f}\right)
\end{aligned}
$$

Amount of $\mathrm{CO}_{2}$ emissions $e_{f_{i}}^{c o m p}$ released from a compressor system involved in process $i$ at factory $f$ as below:

$e_{f_{i}}^{c o m p}=\sum_{i=1}^{\Pi_{f}}\left(0.689 \frac{q_{f_{i}}^{r}}{\mathfrak{R}_{f i} \mu_{f i}} \frac{N_{f_{i}}^{c o m p}}{\rho_{f_{i}}^{\text {comp }}} v_{f_{i}}^{\text {comp }} n_{f_{i}}^{\text {mach }}\right)$, where 0.689 is the emission factor for the

electricity

Amount of $\mathrm{CO}_{2}$ emissions $e_{w}$ released from warehouse $w$ is calculated as below:

$$
e_{w}=0.989 \sum_{w=1}^{W}\left(N_{w}^{c o n d} n_{w}^{c o n d} \frac{q_{f w}^{m p}}{\wp_{w}}+N_{w}^{b u l b} \times n_{w}^{b u l b} \times \frac{q_{f w}^{m p}}{\wp_{w}}\right)
$$

Thus, equation 29 is given as follows: 


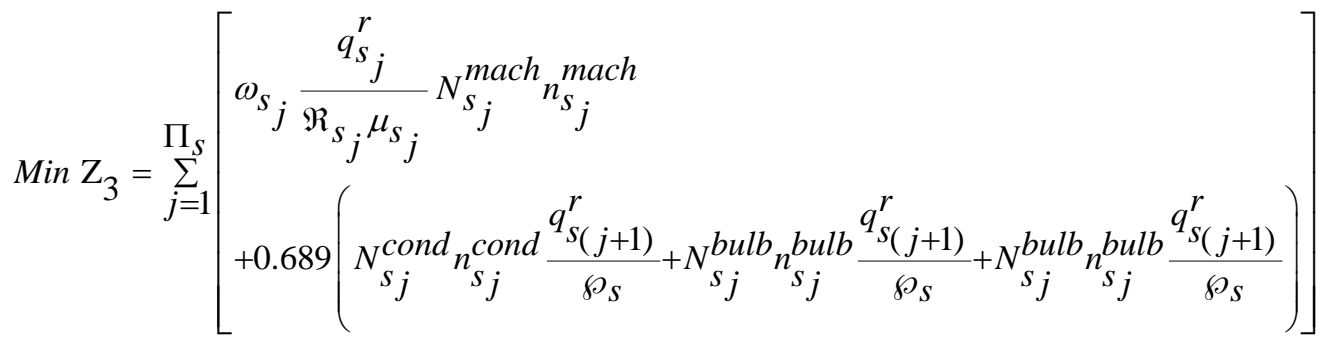

$$
\begin{aligned}
& +\sum_{s=1}^{S} \sum_{f=1}^{F}\left(\omega_{s f}^{t} \frac{q_{s f}^{r}}{V} T_{s f}\right)+\sum_{f=1}^{F} \sum_{w=1}^{W}\left(\omega_{f w}^{t} \frac{q_{f w}^{m p}}{V} T_{f w}\right)
\end{aligned}
$$

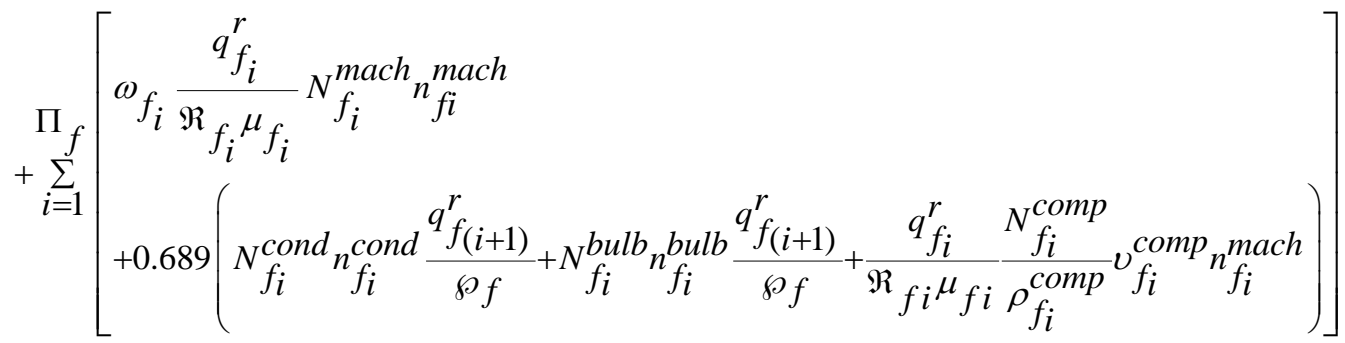

$$
\begin{aligned}
& +0.689 \sum_{w=1}^{W}\left(N_{w}^{\text {cond }} n_{w}^{\text {cond }} \frac{q_{f w}^{m p}}{\wp_{w}}+N_{w}^{b u l b} \times n_{w}^{b u l b} \times \frac{q_{f w}^{m p}}{\wp_{w}}\right)
\end{aligned}
$$

Where, the $\mathrm{CO}_{2}$ emission factor $\omega_{s_{j}}, \omega_{f_{i}}, \omega_{w}$ and $\omega_{s f}^{t}$ is listed in Table 1 (EPA, 2008); Nujoom et al., 2016).

Table. 1. Amount $\mathrm{CO}_{2}$ emission factor per kWh and Per mile

\begin{tabular}{ccc}
\hline Energy source & $\begin{array}{c}\text { Emission factor } \omega_{s}, \omega_{f_{i}} \text { and } \omega_{w} \\
(\mathrm{~kg} / \mathrm{kWh})\end{array}$ & $\begin{array}{c}\text { Emission factor } \omega_{s f, f w}^{t} \text { for truck } \\
(\mathrm{kg} / \mathrm{mile})\end{array}$ \\
\hline $\begin{array}{c}\text { Oil as indirect energy source to } \\
\text { generate electricity }\end{array}$ & 0.6895 & 0.420 \\
\hline
\end{tabular}

\subsection{Constraints}

Equations 41 and 42 limit that the amount of raw material transported from supplier $s$ to factory $f$ and products transported from factory $f$ to warehouse $w$ cannot exceed their capacity.

$q_{s f}^{r} \leq C a_{s}$

$q_{f w}^{m p} \leq C a_{f}$ 
Equations 43 and 44 ensure that the demands of factory $f$ and warehouse $w$ are fulfilled, respectively.

$q_{s f}^{r} \geq D_{f}$

$q_{f w}^{m p} \geq D_{w}$

Equations 45 and 46 ensure that quantity of raw materials of the first process tasks $j$ and $i$ must be bigger

than or equal to quantity of materials of the next process task $(j+1)$ and $(i+1)$ in supplier $s$ and factory $f$, respectively.

$$
\begin{aligned}
& \left(1-\Psi_{s_{j}}\right) q_{S_{j}}^{r} \geq q_{S(i+1)}^{r} \\
& \left(1-\Psi_{f_{i}}\right) q_{f_{(i+1)}}^{r} \geq q_{f_{(i+1)}}^{r}
\end{aligned}
$$

Equations 47 and 48 are defined in which the quantity of machines in process tasks $j$ in supplier $s$ and $i$ in factory $f$ (being served by one air-conditioning unit) is limited to be less than or equal to the quantity of air-conditioning units in this process respectively.

$$
\begin{gathered}
\Phi_{s_{j}}^{\text {cond } n_{s_{j}}^{\text {cond }} \geq n_{s_{j}}^{\text {mach }}} \\
\Phi_{f_{i}}^{\text {cond }} n_{f_{i}}^{\text {cond }} \geq n_{f_{i}}^{\text {mach }}
\end{gathered}
$$

Equations 49 and 50 are defined that the number of light bulbs, which serve all the machines involved in process tasks $j$ in supplier $s$ and $i$ in factory $f$, must be greater than or equal to the number of machines involved in this process respectively.

$$
\begin{aligned}
& n_{s_{j}}^{\text {bulb }} \geq \varphi_{s_{j}}^{\text {bulb }} n_{s_{j}}^{\text {mach }} \\
& n_{f_{i}}^{\text {bulb }} \geq \varphi_{f_{i}}^{\text {bulb }} n_{f_{i}}^{\text {mach }}
\end{aligned}
$$

Equations 51 and 52 ensure that the quantity of products being covered using one air-conditioning unit and one lighting bulb in warehouse $w$, respectively. 
$\Gamma_{w}^{\text {cond }} n_{w}^{\text {cond }} \geq q_{f w}^{m p}$

$\lambda_{w}^{b u l b} n_{w}^{b u l b} \geq q_{f w}^{m p}$

Equation 53 is a non-negativity constraint for the quantity of materials shipped from supplier $s$ to factory $f$ and for products shipped from factory $f$ to warehouse $w$.

$q_{s j}^{r}, q_{s f}^{r}, q_{f_{i}}^{r}, q_{f w}^{m p} \geq 0$

Equations 54 and 55 are defined that the manufacturing rate of process tasks $j$ and $i$ in supplier $s$ and factory $f$ must be greater than or equal to the quantity of materials involved in the next process task $(j+1)$ and $(i+1)$ in supplier $s$ and factory $f$, respectively.

$\mathfrak{R}_{s_{j}} n_{s_{j}}^{\text {mach }} \geq q_{S_{(i+1)}}^{r}$

${ }^{\Re} f_{i}^{n} f_{i}^{m a c h} \geq q f_{(i+1)}$

Where, equations 41-46 and 53 are quantity constraints; and equations 47-52, 54 and 55 are constraints in numbers of machines, air-conditioning units and lighting bulbs.

\section{Optimization methodology}

In this section, two optimization approaches i.e. an integrated DEMATEL-E-constraint approach and goal programming approach were applied to reveal non-inferior solutions based on the developed three objectives optimization model. The DEMATEL algorithm was used to determine the most important objective to be kept as an objective function in implementing the $\varepsilon$-constraint approach. Briefly, the two approaches were utilized as described below:

\section{1.e-constraint}


Based on the $\varepsilon$-constraint approach, the developed multi-objective model is re-presented as a mono objective model by adding constraints; the higher priority objective (i.e., total energy consumption) is considered to be an objective function (equation 56) and the other two objective functions (i.e., the total cost and the total $\mathrm{CO}_{2}$ emissions) are moved to $\varepsilon$-based constraints. In this research, it is noteworthy that the DEMATEL algorithm was used to determine the most important objective to be kept as an objective function (see the next subsection 4.1.1). The solution objective function $\mathrm{Z}$ is expressed as follows (Chankong \& Haimes, 1983):

\section{$\operatorname{Min} Z_{2}$}

Eq. (55) is subject to the following constrains:

$$
\begin{aligned}
& Z_{1} \leq \varepsilon_{1} \\
& \left(Z_{1}\right)^{\min } \leq \varepsilon_{1} \leq\left(Z_{1}\right)^{\max } \\
& Z_{3} \leq \varepsilon_{2} \\
& \left(Z_{3}\right)^{\min } \leq \varepsilon_{2} \leq\left(Z_{3}\right)^{\max }
\end{aligned}
$$

And additional constraints including (equation. 41-55)

Equation 57 limits the value of the first objective to be less than or equal to $\varepsilon_{1}$ which changes between the minimum value and the maximum value for objective function one (equation 58). Equation 59 restricts the value of the third objective function to be less than or equal to $\varepsilon_{2}$ which gradually varies between the minimum value and the maximum value for objective function three (equation 60).

\subsubsection{Decision-making trial and evaluation laboratory (DEMATEL)}

DEMATEL is a multi-attribute decision making algorithm used to determine the weights of attributes and to examine the relationship between different variables of a complicated system. The implementation of DEMATEL includes the following steps (Tzeng et al., 2007):

Step 1: Generate the linguistic evaluation decision matrix based on decision makers 'expert. In this research the linguistic evaluation and its correspondence quantitative scale are shown in Table 2. 
Step 2: Generate the quantitative pairwise compassion among the considered attribute by converting the linguistic evaluation obtained from Step 1 using the quantitative scale shown in Table 2.

$A_{i j}=\left[\begin{array}{lll}r_{11} & r_{12} & r_{1 j} \\ r_{21} & r_{22} & r_{2 j} \\ \cdot & \cdot & \cdot \\ \cdot & \cdot & \cdot \\ r_{i 1} & r_{i 2} & r_{i j}\end{array}\right]$

Where $A_{i j}$ represents a pairwise decision matrix, in which the element $r_{i j}$ denotes the level to which the $i$ th attribute influence the $j$ th attribute.

Step 3: After generating the pairwise decision matrix, generate the normalized direct-relation matrix $N$ which can be generated using Eq. (61).

$$
N=A \cdot K
$$

Where

$$
K=\frac{1}{\max _{1<i<n}\left(\sum_{j \in n} r_{i j}\right)} ; i, j=1, \ldots, n
$$

Step 4: Generate the total-relation matrix $T$ using Eq. 63, in which $I$ denotes the identity matrix. The matrix $T$ reveals the total relationship between each pair of decision attribute.

$$
T=N(I-N)^{-1}
$$

Step 5: Sum rows and columns of matrix $T$ using Eqs. 64 and 65. These two summations are represented by $\mathrm{D}$ and $\mathrm{R}$ vectors.

$$
\begin{aligned}
& D_{i}=\left[\sum_{j \in n} t_{i j}\right]_{n x 1} ; i=1,2, \ldots, n \\
& R_{j}=\left[\sum_{i \in n} t_{i j}\right]_{1 x n} ; j=1,2, \ldots, n
\end{aligned}
$$


Step 6: Define a threshold value $a$. Matrix $T$ shows information on how one attribute influences another, it thus becomes required for the decision makers to define a threshold value $a$ for elucidating the structural relation among attributes while simultaneously keeping the intricacy of the entire system to a convenient level. An influence relationship between two attributes is excluded from the evaluation if their correlation value in matrix $T$ is smaller than $a$ and only the effects greater than the set $a$ value are chosen and shown in the digraph. In this work, the threshold value $a$ is determined from the average of the values in matrix $T$ using Eq. (66), where $\mathrm{N}$ is the total number of values in matrix $T$.

$$
a=\frac{\sum_{i \in n} \sum_{j \in n} t_{i j}}{N}
$$

Step 7: Build the relationship table by summing $D$ and $R$ and subtracting $D$ from $R$ in which $D+R$ vector reveals how much importance the criterion has. The $D-R$ vector divide the attribute into the causal and effect groups. Generally, a positive value of $D$ - $R$ refers to the attributes that belongs to the causal group and if a negative value $D-R$ refers to the attributes that belongs to the effect group.

Step 8: Use Eq. 67 to determine the importance weight for each attribute by normalizing the $D+R$ vector in which the sum of normalized weights equals to 1 .

$$
w_{i}=\frac{(D+R)_{i}}{\left(\sum_{i \in n}(D+R)_{i}\right)} ; i=1,2, \ldots, n
$$

Table. 2. Linguistic variables and correspondence scales used for evaluating the three objectives 
Lo influence (LI)

High influence (HI)

\subsection{Goal Programming}

The purpose of the goal programming approach is to find a solution that minimizes undesirable deviations between the objective functions and their corresponding goals (Charnes et al., 1955; Mohammed et al., 2017). The equivalent solution formulas are presented as follows:

\section{Min Z}

$$
\frac{\varsigma^{1}}{\mathrm{G}^{1}} \leq Z
$$

$$
\frac{v^{2}}{G^{2}} \leq Z
$$

$$
\frac{v^{3}}{G^{3}} \leq Z
$$

The equivalent objective functions are presented as follows. 
Min $\mathrm{Z}_{1}=C_{S}^{\text {land }}+C_{S}^{\text {building }}+C_{S}^{\text {equipment }}+C_{S}^{\text {services }}+C_{S}^{\text {saleries }}$

$+C_{f}^{\text {land }}+C_{f}^{\text {building }}+C_{f}^{\text {equipment }}+C_{f}^{\text {services }}+C_{f}^{\text {saleries }}+C_{w}^{\text {land }}$

$+C_{w}^{\text {building }}+C_{w}^{\text {equipment }}+C_{w}^{\text {services }}+C_{w}^{\text {saleries }}+\sum_{j=1}^{\prod_{s}}\left(C_{s_{j}}^{\text {mach }} n_{s_{j}}^{\text {mach }}\right)$

$+\sum_{i=1}^{\Pi_{f}}\left(C_{f_{i}}^{\text {mach }} n_{f_{i}}^{\text {mach }}\right)+\sum_{j=1}^{\Pi_{s}}\left(C_{s_{j}}^{\text {cond }} n_{s_{j}}^{\text {cond }}\right)+\sum_{j=1}^{\Pi_{f}}\left(C_{f_{i}}^{\text {cond }} n_{f_{i}}^{\text {cond }}\right)+\sum_{w=1}^{W}\left(C_{w}^{\text {cond }} n_{w}^{\text {cond }}\right)$

$+\sum_{j=1}^{\Pi_{S}}\left(C_{s_{j}}^{\text {bulp }} n_{s_{j}}^{\text {bulb }}\right)+\sum_{i=1}^{\Pi_{f}}\left(C_{f_{i}}^{\text {bulp }} n_{f_{i}}^{\text {bulb }}\right)+\sum_{w=1}^{W}\left(C_{w}^{b u l p} n_{w}^{\text {bulb }}\right)+\sum_{s=1}^{S} \sum_{f=1}^{F} C_{S}^{r} q_{s f}^{r}$

$+\sum_{f=1}^{F} \sum_{w=1}^{W} C_{f}^{m p} q_{f w}^{m p}+\sum_{s=1}^{S} \sum_{f=1}^{F} C_{s f}^{t} \frac{q_{s f}^{r}}{V} T_{s f}+\sum_{f=1}^{F} \sum_{w=1}^{W} C_{f w}^{t} \frac{q_{f w}^{m p}}{V} T_{f w}+\sum_{f=1}^{F} \sum_{w=1}^{W} C_{w}^{I} q_{f w}^{m p}+\varsigma^{1}-v^{1}=\mathrm{G}^{1}$

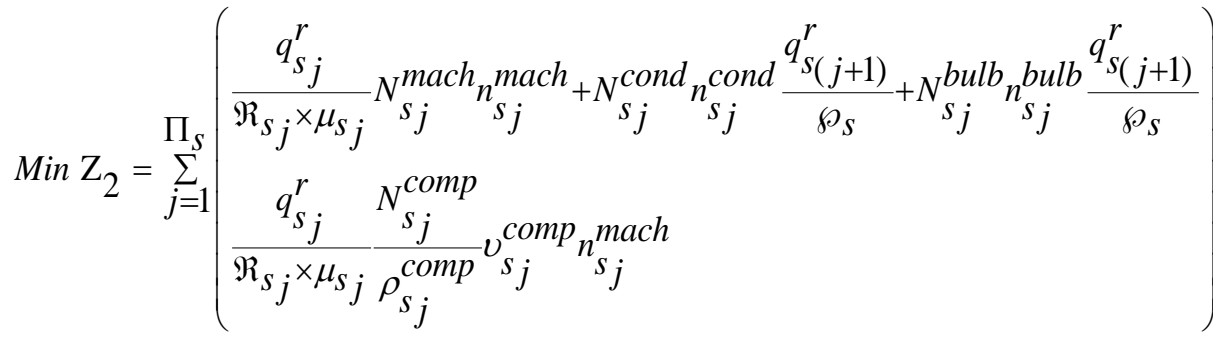

$$
\begin{aligned}
& +\sum_{i=1}\left(\begin{array}{l}
\frac{q_{f_{i}}^{r}}{\Re_{f_{i}} \times \mu_{f_{i}}} N_{f_{i}}^{\text {mach }} n_{f_{i}}^{\text {mach }}+N_{f_{i}}^{\text {cond }} n_{f_{i}}^{\text {cond }} \frac{q_{f_{(i+1)}}^{r}}{\wp f}+N_{f_{i}}^{\text {bulb }} n_{f_{i}}^{\text {bulb }} \frac{q_{f_{(i+1)}}^{r}}{\wp f} \\
+\frac{q_{f_{i}}^{r}}{\Re_{f_{i}} \times \mu_{f_{i}}} \frac{N_{f_{i}}^{\text {comp }}}{\rho_{f_{i}}^{\text {comp }}} v_{f_{i}}^{\text {comp }} n_{f_{i}}^{\text {mach }}
\end{array}\right) \\
& +\sum_{w=1}^{W}\left(N_{w}^{c o n d} n_{w}^{c o n d} \frac{q_{w}^{m p}}{\wp_{w}}+N_{w}^{b u l b} n_{w}^{b u l b} \frac{q_{f w}^{m p}}{\wp_{w}}\right)+\varsigma^{2}-v^{2}=\mathrm{G}^{2}
\end{aligned}
$$




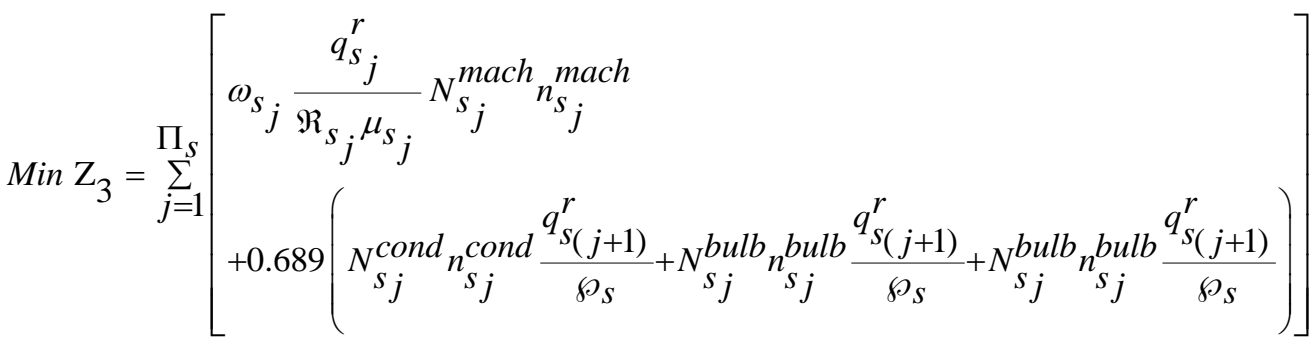

$$
\begin{aligned}
& +\sum_{S=1}^{S} \sum_{f=1}^{F}\left(\omega_{s f}^{t} \frac{q_{s f}^{r}}{V} T_{s f}\right)+\sum_{f=1}^{F} \sum_{w=1}^{W}\left(\omega_{f w}^{t} \frac{q_{f w}^{m p}}{V} T_{f w}\right)
\end{aligned}
$$

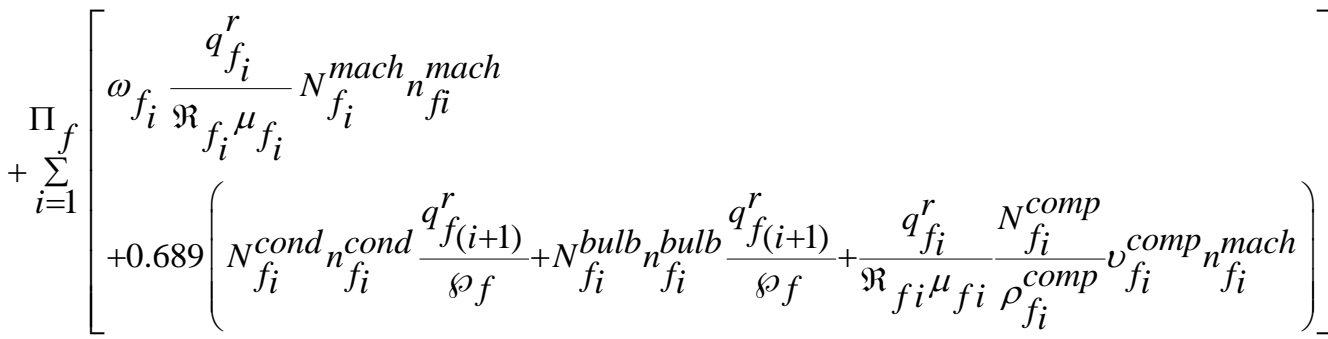

$$
\begin{aligned}
& +0.689 \sum_{w=1}^{W}\left(N_{w}^{\text {cond }} n_{w}^{\text {cond }} \frac{q_{f w}^{m p}}{\wp_{w}}+N_{w}^{b u l b} \times n_{w}^{b u l b} \times \frac{q_{f w}^{m p}}{\wp_{w}}\right)+\varsigma^{3}-v^{3}=\mathrm{G}^{3}
\end{aligned}
$$

Where

$$
\begin{aligned}
& \mathrm{G}^{1} \quad \text { goal of the objective } 1 \\
& \mathrm{G}^{2} \quad \text { goal of the objective } 2 \\
& \mathrm{G}^{3} \quad \text { goal of the objective } 3 \\
& \varsigma^{1} \quad \text { negative deviation variable of the objective } 1 \\
& \varsigma^{2} \quad \text { negative deviation variable of the objective } 2 \\
& \varsigma^{3} \quad \text { negative deviation variable of the objective } 3 \\
& v^{1} \quad \text { positive deviation variable of the objective } 1 \\
& v^{2} \quad \text { positive deviation variable of the objective } 2 \\
& v^{3} \quad \text { positive deviation variable of the objective } 3
\end{aligned}
$$

Subject to an additional non-negativity restriction: 


\section{Application and evaluation: a real case study}

This section presents an application of the developed multi-objective optimization model to evaluate its applicability in a real case study. The SMS includes three facilities (supplier $s$, factory $f$ and warehouse $w$ ), and both facilities supplier $s$ and factory $f$ have a number of processing tasks in which each process task may has a number of machines, number of air-conditioning units and number of lighting bulbs. Each of those equipment has consumption of energy, releases an amount of $\mathrm{CO}_{2}$ emissions and has mass inputs with different specifications. Table 3 shows the manufacturing process with the symbols representing each task of a manufacturing process for the production of plastic and woven sacks inside supplier and factory. Table 4 lists the collected data were taken from a manufacturing system which includes three facilities ( 1 supplier, 1 factory and 1 warehouse) used for producing plastic and woven sacks. In this case, the production line is powered by electricity which is generated using oil as indirect source of energy. The developed multi-objective optimization problem was solved using LINGO $^{11}$ software. The study was conducted by analysing the total cost for establishing these facilities, the energy consumption and the amount of $\mathrm{CO}_{2}$ emissions towards a SMS design.

Table. 3. Processes tasks related to a plastic and woven sacks manufacturer

\begin{tabular}{ccc}
\hline Tasks & Description & predecessors \\
\hline A & Gas-phase & None \\
B & Converted the gas to liquid & A \\
D & Converted the liquid to powder & B \\
H & Converted powder to pellets & G \\
R.M & Raw material (polypropylene) & R.M \\
G & Extruding the Polypropylene to make stands & K \\
W & Weaving the stands into rolls of sacks & H \\
L & Laminating the rolls & L \\
P & Printing and branding & P \\
C & Cutting the rolls into bags & C \\
K & Inserts and smoothes out blown film into the bags & M \\
S & Blown film sewn into bag & Y \\
Z & End product compressed & $\mathrm{Z}$
\end{tabular}


Tabl.4. Data related to the case study

\begin{tabular}{|c|c|c|}
\hline \multicolumn{3}{|c|}{ Facilities } \\
\hline Supplier $s$ & Factory $f$ & Warehouse $w$ \\
\hline$C_{S}^{e S}(\mathrm{GBP}): 100000$ & $C_{f}^{e s}(\mathrm{GBP}): 100000$ & $C_{w}^{e s}$ (GBP): 55000 \\
\hline $\begin{array}{l}C_{s_{j}}^{m a c h}(\mathrm{GBP}): 7000,7000,7000,7000, \text { where } \\
j \in\left\{1,2, \ldots ., m_{s}\right\}\end{array}$ & $\begin{array}{l}C_{f_{i}}^{m a c h}(\mathrm{GBP}): 5000,3000,4000,3000,3000, \\
100,200,2000, \text { where } i \in\left\{1,2, \ldots ., m_{f}\right\}\end{array}$ & \\
\hline$C_{s_{j}}^{c o n d}(\mathrm{GBP}): 1000,1000,1000,1000$ & $\begin{array}{l}C_{f_{i}}^{\text {cond }}(\mathrm{GBP}): 1000,1000,1000,1000,1000 \\
1000,1000,1000\end{array}$ & $C_{w}^{c o n d}(\mathrm{GBP}): 700$ \\
\hline$C_{s_{j}}^{b u l p}(\mathrm{GBP}): 50,50,50,50$ & $C_{f_{i}}^{b u l p}{ }_{(\mathrm{GBP}): 50,50,50,50,50,50,50,50}$ & $C_{w}^{b u l b}(\mathrm{GBP}): 50$ \\
\hline$C_{S}^{r}(\mathrm{GBP} / \mathrm{kg}): 2$ & $C_{f}^{m p}(\mathrm{GBP} / \mathrm{kg}): 3$ & $C_{w}^{I}(\mathrm{GBP} / \mathrm{kg}): 2$ \\
\hline$C_{s f}^{t}(\mathrm{GBP} / \mathrm{mile}): 2$ & $C_{f w}^{t}(\mathrm{GBP} / \mathrm{mile}): 2$ & \\
\hline$T_{s f}\left(\right.$ mile): $50, V_{(\mathrm{kg}): 20000}$ & $T_{f w}($ mile): $10, V=20000$ & ----------- \\
\hline$C a_{S}$ (kg/month): 1000000 & $C a_{f}$ (kg/month): 990,000 & $C a_{w}$ (kg/month): 900000 \\
\hline ------------- & $D_{f}$ (kg/month): 850000 & $D_{w}$ (kg/month): 850000 \\
\hline$\Pi_{s}=4$ process & $\Pi_{f}=8$ process & ------------- \\
\hline $\begin{array}{l}\mathfrak{R}_{s_{j}}(\mathrm{~kg} / \mathrm{h}): 1976,1936,1932 \text { and } 1929, \text { where } \\
j \in\left\{1,2, \ldots ., m_{s}\right\}\end{array}$ & $\begin{array}{l}\mathfrak{R}_{f_{i}}(\mathrm{~kg} / \mathrm{h}): 1852,1815,1742,1716,1699,1665, \\
1660 \text { and } 1643, \text { where } i \in\left\{1,2, \ldots ., m_{f}\right\}\end{array}$ & \\
\hline$\mu_{s_{j}}(\%): 80$ for all machines & $\mu_{f_{i}}(\%): 80$ for all machines & ----------- \\
\hline$\Psi_{S_{j}}(\%): 0.03,0.02,0.002,0.15$ & $\Psi_{f_{i}}(\%): 0.02,0.04,0.015,0.01,0.02,0.003,0.01$ & ------------- \\
\hline$N_{s_{j}}^{m a c h}(\mathrm{kw}): 700,500,300,600$ & $N_{f_{i}}^{m a c h}(\mathrm{kw}): 200,20,7,40,7,0,0.8,4$ & ------------- \\
\hline$N_{s_{j}}^{\operatorname{comp}}{ }_{(\mathrm{kw}): 0}$ & $N_{f_{i}}^{\text {comp }}{ }_{(\mathrm{kw}): 200}$ & ------------- \\
\hline$\rho_{s_{j}}^{c o m p}\left(\mathrm{~m}^{3} / \mathrm{h}\right): 0$ & $\rho_{f_{i}}^{\operatorname{comp}}\left(\mathrm{m}^{3} / \mathrm{h}\right): 666$ & ------------- \\
\hline$v_{s_{j}}^{\operatorname{comp}}\left(\mathrm{m}^{3} / \mathrm{h}\right): 0$ & $v_{f_{i}}^{c o m p}\left(\mathrm{~m}^{3} / \mathrm{h}\right): 5,4,13,0,7,5,20,0,0,0$ & ---- \\
\hline$N_{s_{j}}^{\text {cond }}(\mathrm{kw}): 2.2$ & $N_{f_{i}}^{\text {cond }}{ }_{(\mathrm{kw}): 2.2}$ & $N_{w}^{c o n d}(\mathrm{kw}): 2.2$ \\
\hline$N_{s_{j}}^{b u l b}$ (kw): 0.4 & $N_{f_{i}}^{b u l b} \quad(\mathrm{kw}): 0.4$ & $N_{w}^{b u l b}$ (kw): 0.4 \\
\hline$\Phi_{S_{j}}^{c o n d}$ (units): $2, \varphi_{S_{j}}^{b u l b}$ (units): 15 & $\Phi_{f_{i}}^{\text {cond }}$ (units): $2, \varphi_{f_{i}}^{b u l b}$ (units): 15 & $\Gamma_{w}^{c o n d}{ }_{(\mathrm{kg}): 1000, \lambda_{w}^{b u l b}}^{\text {ckg): } 500}$ \\
\hline$\wp_{s}(\mathrm{~kg}): 950000$ & $\wp_{f}(\mathrm{~kg}): 840000$ & $\wp_{w}$ (units): $9,032,258$ \\
\hline$\omega_{s_{j}}(\mathrm{~kg} / \mathrm{kWh}): 0.6895$ & $\omega_{f_{i}}(\mathrm{~kg} / \mathrm{kWh}): 0.6895$ & $\omega_{w}(\mathrm{~kg} / \mathrm{kWh}): 0.6895$ \\
\hline$\omega_{s f}^{t}(\mathrm{~kg} / \mathrm{mile}): 0.420$ & $\omega_{f w}^{t}(\mathrm{~kg} / \mathrm{mile}): 0.420$ & ----------- \\
\hline
\end{tabular}


Two optimization approaches (i.e., an integrated DEMATEL- $\varepsilon$-constraint and goal programming) were used to obtain two sets of non-inferior solutions derived from co-optimizing the three objectives in minimizing total $\operatorname{cost} Z_{1}$, energy consumption $Z_{2}$, and $\mathrm{CO}_{2}$ emissions $Z_{3}$. The two optimization approaches were implemented as follows:

\subsection{1. $\quad \varepsilon$-constraint:}

As mentioned previously (see section 4.1), the most important objective has to be kept as an objective function and the other two objectives are shifted to the constraints. In view of the fact that determining the most important objective is actually an intricate multi-criteria decision making problem, thus DEMATEL algorithm was used. First, two decision makers (DM1 and DM2) from engineering and production department were invited to perform the linguistic evaluation of three objectives to generate the comparison matrix of DEMATEL and, hence, determine the importance weights of three objectives. The pairwise comparison was generated based on a $0,1,2,3$ and 4 scale as illustrated previously in Table 2. Table 5 shows the linguistic evaluation of the three objectives from the two decision makers 'perspective. Table 6 shows the importance weights (IW) of the three objectives in addition to $(D+R)$ and $(D-R)$ values. The latter values show the degree of total influence levels and the degree of net influence levels respectively, where the positive values indicate that it will influence other objectives more than any other objectives influences it. As sown in Table 6, the objective of minimizing the total energy consumption revealed the largest net influence level followed by the total cost and total $\mathrm{CO}_{2}$ emissions, respectively. As shown in Table 6, objective of minimizing the energy consumption has the highest importance weight $(0.365354387)$ followed by minimizing the total cost $(0.338070335)$ and minimizing the total $\mathrm{CO}_{2}$ emissions (0.296573326), respectively. In other words, these results proved that objective two is the most important objective based on its influence on other two objectives. Thus, the minimization of energy consumption was kept as an objective function and minimization of total cost and $\mathrm{CO}_{2}$ emissions were shifted to the constraints. Table 7 , presents the non-inferior solutions obtained by an assignment of $\varepsilon$-values from $20,781,782$ to $26,000,000$ for objective one and from $103.75 \times 10^{9}$ to $158.75 \times 10^{9}$ for objective three. It can 
be noted in Table 7 that the values of objective one and three are highly corresponding to the assigned values of $\varepsilon_{1}$ and $\varepsilon_{2}$ which vary between the minimum and maximum value for objectives one and three, respectively. As an example, solution 1 obtained by an assignment of $\varepsilon_{1}=20,781,782$, and $\varepsilon_{2}=103.75 \times 10^{9}$ accordingly, the minimum total manufacturing system cost is $20,500,000 \mathrm{GBP}$, the minimum total energy consumption is $2,357,288 \mathrm{kWh}$ and the minimum $\mathrm{CO}_{2}$ emissions is $103748 \times 10^{6} \mathrm{~kg}$.

\subsubsection{Goal programming:}

The goal programming approach was implemented by assigning five different goals for the three criteria. These goals were obtained by solving the three objectives individually to obtain the ideal value. Table 8 shows the solution results obtained using the goal programming approach; this includes five non-inferior solutions. For instance, solution number 1 leads to a minimum total cost of 22,183,564 GBP, a minimum total energy consumption of $2,800,500 \mathrm{kWh}$ and a minimum total $\mathrm{CO}_{2}$ emission of $107493 \times 10^{6} \mathrm{~kg}$.

Table. 5. Linguistic evaluation of the three objectives based on DM1 and DM2

\begin{tabular}{llll}
\hline Decision maker & & DM1 & \\
\hline Objective & Cost & Energy & $\mathrm{CO}_{2}$ \\
\hline $\mathrm{C}$ & $\mathrm{NI}$ & $\mathrm{H}$ & $\mathrm{VH}$ \\
$\mathrm{EI}$ & $\mathrm{LI}$ & $\mathrm{NI}$ & $\mathrm{LI}$ \\
$\mathrm{EC}$ & $\mathrm{VH}$ & $\mathrm{VH}$ & $\mathrm{NI}$ \\
\hline Decision maker & & $\mathrm{DM} 2$ & \\
\hline Objective & Cost & Energy & $\mathrm{CO}_{2}$ \\
\hline $\mathrm{C}$ & $\mathrm{NI}$ & $\mathrm{H}$ & $\mathrm{H}$ \\
EI & $\mathrm{LI}$ & $\mathrm{NI}$ & $\mathrm{MI}$ \\
EC & $\mathrm{H}$ & $\mathrm{VH}$ & $\mathrm{NI}$ \\
\hline
\end{tabular}

Table. 6. Total and net influence levels and importance weights for the three objectives 


\begin{tabular}{c|ccccc}
\hline Objective & $\mathrm{D}$ & $\mathrm{R}$ & $\mathrm{D}+\mathrm{R}$ & $\mathrm{D}-\mathrm{R}$ & Weight \\
\hline $\mathrm{C}$ & 2.7863 & 2.0048 & 4.7911 & 0.7814 & 0.338070335 \\
$\mathrm{EI}$ & 1.3040 & 2.8990 & 4.2030 & -1.5951 & 0.296573326 \\
$\mathrm{EC}$ & 2.9957 & 2.1821 & 5.1778 & 0.8136 & 0.365354387 \\
\hline
\end{tabular}

Table.7. Non-inferior solutions via the integrated DEMATEL-E-Constraint approach

\begin{tabular}{cccccc}
\hline Solution & \multicolumn{2}{c}{ Assigned $\varepsilon$ values } & \multicolumn{3}{c}{ Objective function solutions } \\
\cline { 2 - 6 } number & $\varepsilon_{1}$ & $\varepsilon_{2}$ & Min $\mathrm{Z}_{1}$ & Min $\mathrm{Z}_{2}$ (Energy) & Min $\mathrm{Z}_{3}\left(\mathrm{CO}_{2}\right)$ \\
& & & $(\mathrm{Cost})$ & $(\mathrm{kWh} / \mathrm{month})$ & $(\mathrm{kg} / \mathrm{month})$ \\
& & & $(\mathrm{GBP})$ & & \\
\hline 1 & 20781782 & $103.75 \times 10^{9}$ & 20500000 & 2357288 & $103748 \times 10^{6}$ \\
2 & 22123925 & $117.5 \times 10^{9}$ & 21879729 & 2557194 & $117498 \times 10^{6}$ \\
3 & 23466068 & $131.25 \times 10^{9}$ & 23239639 & 2842852 & $131248 \times 10^{6}$ \\
4 & 24808211 & $145 \times 10^{9}$ & 24640700 & 3128510 & $144998 \times 10^{6}$ \\
5 & 26000000 & $158.75 \times 10^{9}$ & 26000000 & 3414168 & $158748 \times 10^{6}$ \\
\hline
\end{tabular}

Table 8.Non-inferior solutions via the goal programming approach.

\begin{tabular}{cccc}
\hline Solution number & \multicolumn{3}{c}{ Objective function solutions } \\
\cline { 2 - 4 } & Min $Z_{l}($ Cost $)$ & Min $Z_{2}$ (Energy) & Min $Z_{3}\left(\mathrm{CO}_{2}\right)$ \\
& $(\mathrm{GBP})$ & $(\mathrm{kWh} /$ month $)$ & $(\mathrm{kg} / \mathrm{month})$ \\
\hline 1 & 22183564 & 2800500 & $107493 \times 10^{6}$ \\
2 & 23623925 & 2818500 & $131298 \times 10^{6}$ \\
3 & 24867850 & 3287852 & $144948 \times 10^{6}$ \\
4 & 26300000 & 3573510 & $158738 \times 10^{6}$ \\
5 & 27401200 & 3814168 & $171493 \times 10^{6}$ \\
\hline
\end{tabular}

Figure 2 shows Pareto fronts among the three objectives obtained via the two solution approaches. Arguably, the two approaches performed well in generating the non-inferior solutions. However, the solutions presented in Figure 2 indicates that the non-inferior solutions generated via the integrated DEMATEL- $\varepsilon$-constraint 
approach; it gives values of the total cost, total energy consumption and total $\mathrm{CO}_{2}$ emissions less than those of the non-inferior solutions generated via the goal programing approach. For instance, the minimum manufacturing system cost under solution 1 using $\varepsilon$-constraint approach is 20,500,000 GBP which is less than the minimum total cost under the goal programing approach $(22,183,564 \mathrm{GBP})$. Figure 2 also indicates that the non-inferior solutions generated via the integrated DEMATEL- $\varepsilon$-constraint approach that gives values of the total energy consumption and the total $\mathrm{CO}_{2}$ emissions less than those of the non-inferior solutions generated via the goal programing approach. As an example, the minimum total energy consumption under solution 1 via the integrated DEMATEL- $\varepsilon$-constraint approach is $2,357,288 \mathrm{kWh}$ which is less than the minimum total energy consumption via the goal programing approach $(2,800,500 \mathrm{kWh})$ and the minimum total $\mathrm{CO}_{2}$ emissions via the integrated DEMATEL- $\varepsilon$-constraint approach is $103748 \times 10^{6} \mathrm{~kg}$ which is less than the minimum $\mathrm{CO}_{2}$ emissions via the goal programing approach $\left(107493 \times 10^{6} \mathrm{~kg}\right)$. Moreover, the solutions generated via the integrated DEMATEL- $\varepsilon$-constraint approach are more stable compared to the solutions obtained by using the goal programming approach.
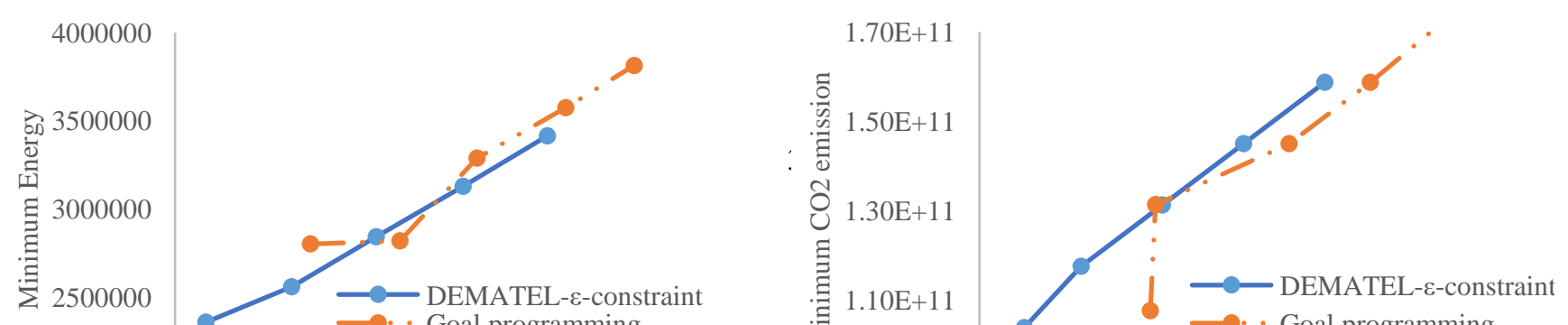


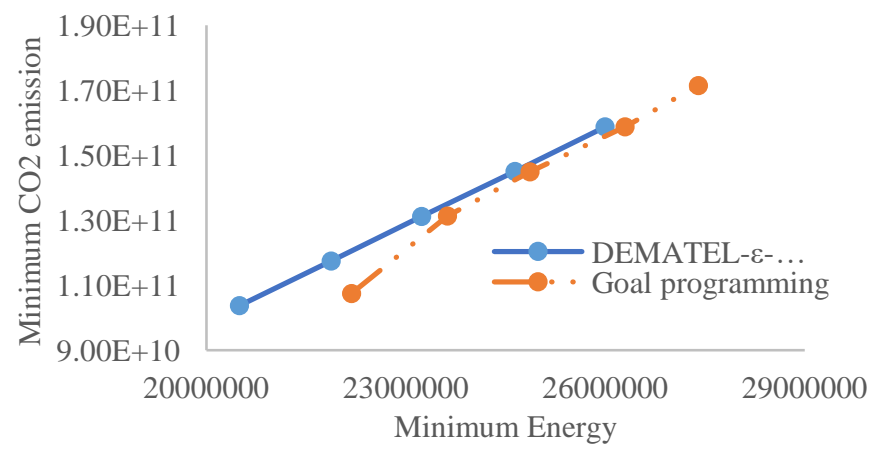

Fig.2. Pareto fronts among the three objectives using the integrated DEMATEL- $\varepsilon$-constraint approach and the goal programing approach

5.2. Determining the number of machines and material flows

Tables 9, 10, 11 and 12 list numbers of machines in the SMS. These solutions are associated with the noninferior solutions generated via the integrated DEMATEL- $\varepsilon$-constraint approach and goal programming approach, respectively. For instance, Table 9 shows the result for solution 1 using the integrated DEMATEL$\varepsilon$-constraint approach which gives the group in numbers of machines that should be involved in process task $j$ at supplier $s n_{s_{j}}^{\text {mach where }} j \in\{1,2,3,4\}$ is $(1,1,1,1)$. Table 10 shows the result for solution 1 using the integrated DEMATEL- $\varepsilon$-constraint approach which gives the group in numbers of machines in process task $i$ in factory $f_{n_{f_{i}}}^{\text {mach }}$ where $i \in\{1,2,3,4,5,6,7,8\}$ is $(4,32,3,5,9,9,35,3)$. 
Table. 9. Number of machines related to process task $j$ in supplier $s$ under the integrated DEMATEL- $\varepsilon$ constraint approach

\begin{tabular}{ccccc}
\hline Solution & \multicolumn{5}{c}{ Numbers of machines in process $j}$, \\
number & \multicolumn{5}{c}{$n_{s_{j}}^{\text {mach }}$ Where $j \in\{1,2,3,4\}}$. \\
& $n_{s 1}$ & $n_{s 2}$ & $n_{s 3}$ & $n_{s 4}$ \\
1 & 1 & 1 & 1 & 1 \\
2 & 1 & 1 & 1 & 1 \\
3 & 2 & 2 & 1 & 1 \\
4 & 2 & 2 & 2 & 1 \\
5 & 2 & 2 & 1 & 2 \\
\hline
\end{tabular}

Table. 10. Numbers of machines related to process task $i$ in factory $f$ under integrated DEMATEL- $\varepsilon$-constraint approach

\begin{tabular}{ccccccccc}
\hline \multirow{2}{*}{ Solution } & \multicolumn{8}{c}{ Numbers of machines in process $i}$, \\
number & \multicolumn{8}{c}{$n_{f_{i}}^{\text {mach }}$ Where $i \in\{1,2,3,4,5,6,7,8\}}$. \\
& \multicolumn{10}{c}{$n_{f 2}$} & $n_{f 3}$ & $n_{f 4}$ & $n_{f 5}$ & $n_{f 6}$ & $n_{f 7}$ & $n_{f 8}$ \\
& 4 & 32 & 3 & 5 & 9 & 9 & 35 & 3 \\
2 & 4 & 32 & 3 & 5 & 10 & 10 & 40 & 3 \\
3 & 4 & 40 & 3 & 5 & 13 & 13 & 60 & 4 \\
4 & 5 & 40 & 4 & 5 & 14 & 14 & 60 & 4 \\
5 & 5 & 45 & 5 & 6 & 16 & 16 & 60 & 5 \\
\hline
\end{tabular}

Table 11 shows the obtained results of solution 1-5 using the goal programing approach. For instance, solution 1 gives the group $(1,2,1,1)$ in numbers of machines, which should be involved in process task $j$ in supplier $s$ $n_{s_{j}}^{\text {mach where }} j \in\{1,2,3,4\}$. Table 12 shows the potential group of number of machines that is in process task $i$ in

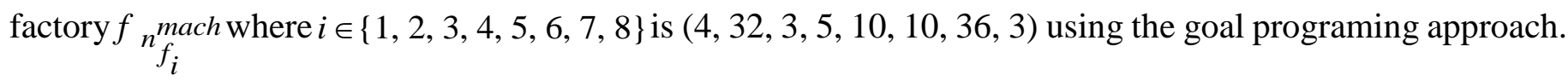

Table. 11. Numbers of machines related to process $j$ in supplier $s$ under the goal programing approach 


\begin{tabular}{|c|c|c|c|c|}
\hline \multirow[t]{2}{*}{$\begin{array}{l}\text { Solution } \\
\text { number }\end{array}$} & \multicolumn{2}{|c|}{ 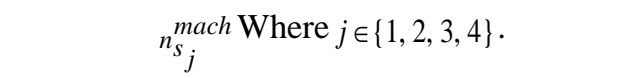 } & & \\
\hline & $n_{s 1}$ & $n_{s 2}$ & $n_{s 3}$ & $n_{s 4}$ \\
\hline 1 & 1 & 2 & 1 & 1 \\
\hline 2 & 1 & 2 & 2 & 1 \\
\hline 3 & 2 & 2 & 1 & 1 \\
\hline 4 & 2 & 2 & 2 & 1 \\
\hline 5 & 2 & 2 & 1 & 2 \\
\hline
\end{tabular}

Table. 12. Numbers of machines related to process $i$ in factory $f$ under the goal programing approach

\begin{tabular}{|c|c|c|c|c|c|c|c|c|}
\hline \multirow{3}{*}{$\begin{array}{l}\text { Solution } \\
\text { number }\end{array}$} & \multicolumn{8}{|c|}{ Numbers of machines in process $i$, } \\
\hline & \multicolumn{8}{|c|}{$n_{f_{i}}^{m a c h}$ Where $i \in\{1,2,3,4,5,6,7,8\}$. } \\
\hline & $n_{f 1}$ & $n_{f 2}$ & $n_{f 3}$ & $n_{f 4}$ & $n_{f 5}$ & $n_{f 6}$ & $n_{f 7}$ & $n_{f 8}$ \\
\hline 1 & 4 & 32 & 4 & 5 & 10 & 10 & 36 & 3 \\
\hline 2 & 4 & 34 & 4 & 6 & 11 & 11 & 42 & 3 \\
\hline 3 & 4 & 40 & 4 & 6 & 14 & 14 & 60 & 4 \\
\hline 4 & 6 & 40 & 4 & 6 & 14 & 14 & 60 & 4 \\
\hline 5 & 6 & 48 & 5 & 6 & 16 & 16 & 60 & 5 \\
\hline
\end{tabular}

Figure 3a, 3b, 3c and 3d, show a comparison among potential groups in numbers of machines that should be established in the manufacturing system based on solution 1 using the integrated DEMATEL- $\varepsilon$-constraint approach and the goal programing approach, respectively. The results in Figure $3 \mathrm{a}$ and $3 \mathrm{~b}$ indicate that the number of machines in process $j$ in supplier $s$ using the integrated DEMATEL- $\varepsilon$-constraint approach is less than the results obtained using the goal programing approach. They indicate that the number of machines needed decreases for process task 2 from 2 to 1 , i.e., from $(1,2,1,1)$ to $(1,1,1,1)$. Figure $3 \mathrm{c}$ and $3 \mathrm{~d}$ indicate that the number of machines in process $i$ in factory $f$ using the integrated DEMATEL- $\varepsilon$-constraint approach is less than the number obtained using the goal programing approach. They indicate that the number of machines needed decreases for process task 3 from 4 to 3, in process task 5 and 6 from 10 to 9 and in process task 7 
from 36 to 35 , i.e., from $(4,32,4,5,10,10,36,3)$ to $(4,32,3,5,9,9,35,3)$. Therefore, the integrated DEMATEL- $\varepsilon$-constraint approach is more efficient than goal programing approach for designing the SMS.

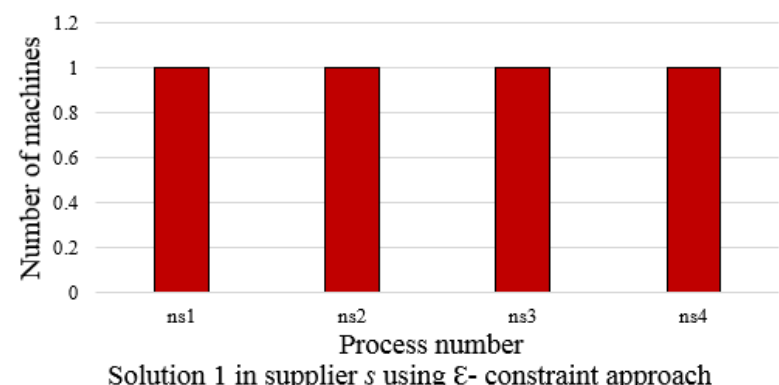

(a)

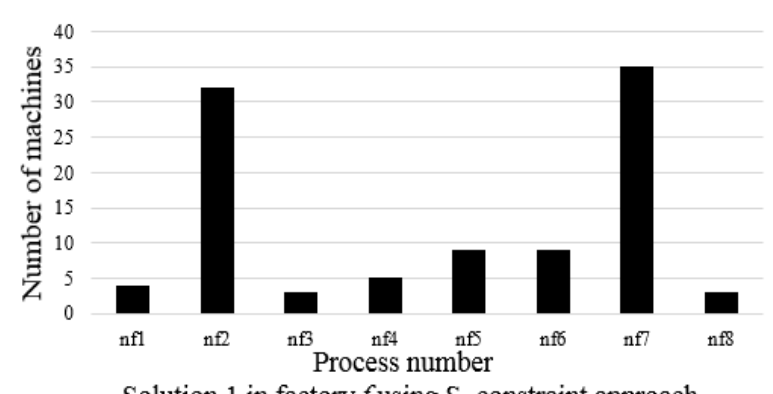

Solution 1 in factory $f$ using $\mathcal{E}$ - constraint approach

(c)

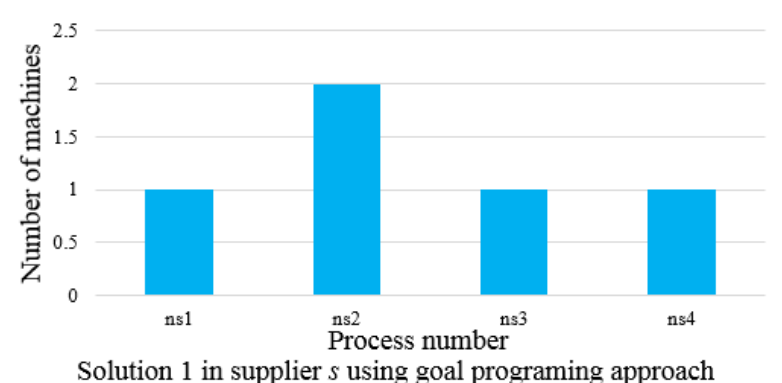

(b)

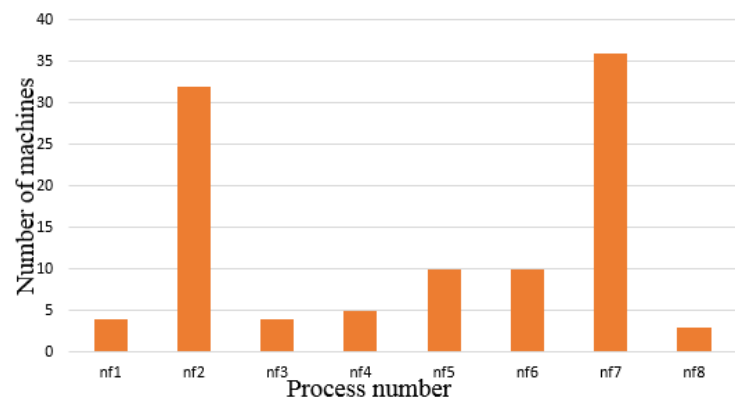

Solution 1 in factory $f$ using goal programing approach (d)

Fig.3. Comparison in numbers of machines at supplier $s$ and factory $f$ associate with solution 3 generated via the integrated DEMATEL- $\varepsilon$-constraint approach and the goal programing approach

\subsection{Selecting the final solution}

Next, decision makers need to select a final solution based on their experts to design the SMS. Based on the latter, solution 3 was selected as the final solution. It gives a total manufacturing system cost of $(23,239,639)$ GBP, an energy consumption of $(2,842,852) \mathrm{kWh}$ and $\mathrm{CO}_{2}$ emissions of $\left(131248 \times 10^{6}\right) \mathrm{kg}$. This solution includes an installation of machines that are required for operating processes task $j$ at supplier $s$ where, $j \in\{1,2,3,4\}$ is $(2,2,1,1)$, and installation of machines that are required in processes task $i$ at factory $f$ where, $i \in\{1,2,3,4,5,6,7,8\}$ is $(4,40,3,5,13,13,60,4)$. Table 13 shows the optimal solutions in quantity of material flows (i) among the machines in process task $j$ at supplier $s$; (ii) from supplier $s$ to factory $f$; and (iii) among the machines in process task $i$ at factory $f$ and (iv) from factory $f$ to warehouse $w$. 
Table.13. The optimal flow of related to the raw material

\begin{tabular}{|c|c|c|c|c|c|c|c|c|c|}
\hline \multicolumn{10}{|c|}{ Supplier $s$} \\
\hline \multirow{2}{*}{$\begin{array}{l}\text { Solution } \\
\text { number }\end{array}$} & \multicolumn{4}{|c|}{$q_{s_{j}}^{r}$ where $j \in\{1,2,3,4\}$} & \multirow{2}{*}{$q_{s f}^{r}$} & - & \multirow[t]{2}{*}{ - } & \multirow{2}{*}{$\begin{array}{l}- \\
-\end{array}$} & \multirow[t]{2}{*}{ - } \\
\hline & $q_{s 1}$ & $q_{s 2}$ & $q_{s 3}$ & $q_{s 4}$ & & - & & & \\
\hline 1 & 955355 & 950050 & 948100 & 946084 & 934570 & - & - & - & \\
\hline 2 & 985500 & 965200 & 963040 & 960084 & 935805 & - & - & - & - \\
\hline 3 & 1000000 & 980000 & 978040 & 976084 & 937040 & - & - & - & - \\
\hline 4 & 1020000 & 1002000 & 996100 & 994084 & 955150 & - & - & - & - \\
\hline 5 & 1045000 & 1027000 & 1009000 & 991100 & 973050 & - & - & - & - \\
\hline \multicolumn{9}{|c|}{ Factory $f$} & Warehouse $w$ \\
\hline \multirow{2}{*}{$\begin{array}{l}\text { Solution } \\
\text { number }\end{array}$} & \multicolumn{8}{|c|}{$q_{f_{i}}^{r}$ where $i \in\{1,2,3,4,5,6,7,8\}$} & $q_{f w p}^{m p}$ \\
\hline & $q_{f l}$ & $q_{f 2}$ & $q_{f 3}$ & $q_{f 4}$ & $q_{f 5}$ & $q_{f 6}$ & $q_{f 7}$ & $q_{f 8}$ & \\
\hline 1 & 934570 & 902423 & 880500 & 842459 & 830550 & 730100 & 600010 & 580200 & $6,238,709$ sacks \\
\hline 2 & 935805 & 909227 & 881567 & 853478 & 842344 & 838459 & 790939 & 600100 & $6,452,688$ sacks \\
\hline 3 & 937040 & 918299 & 889824 & 868344 & 850660 & 840467 & 835940 & 831540 & $8,941,290$ sacks \\
\hline 4 & 955150 & 928300 & 904824 & 883344 & 865660 & 855467 & 850940 & 846540 & $9,102,580$ sacks \\
\hline 5 & 973050 & 940200 & 919700 & 898400 & 883660 & 870500 & 868940 & 864499 & $9,295,688$ sacks \\
\hline
\end{tabular}

Table 14 shows the result of solution 3 related to the numbers of machines and the quantity of materials that are required in the SMS. Figure 4 shows the optimal design of the SMS based on solution 3, which was obtained with $\varepsilon_{1}=23,466,068$, and $\varepsilon_{2}=131.25 \times 10^{9}$ that yields the optimal total cost of $23,239,639$ GBP, the energy consumption of $2,842,852 \mathrm{kWh}$ and the $\mathrm{CO}_{2}$ emissions of $131,248 \times 10^{6} \mathrm{~kg}$. 
Table.14. Numbers of machines and quantity of materials related to solution 3 see (Table 7).

\begin{tabular}{|c|c|c|}
\hline \multicolumn{3}{|c|}{ The optimal solution for supplier $s$} \\
\hline $\begin{array}{c}\text { Process } \\
\text { number } \\
j\end{array}$ & $\begin{array}{l}\text { Number of machines in process } j \\
\qquad n_{s_{j}}^{\text {mach (units) }}\end{array}$ & $\begin{array}{l}\text { Quantity of materials in process } j \\
\qquad q_{s_{j}}^{r}(\mathrm{~kg})\end{array}$ \\
\hline 1 & 2 & 1000000 \\
\hline 2 & 2 & 980000 \\
\hline 3 & 1 & 978040 \\
\hline 4 & 1 & 976084 \\
\hline mass of $n$ & $\begin{array}{l}\text { rial }(\mathrm{kg}) \text { transported from supplier } s \\
\text { to factory } f \\
q_{s f}^{r}(\mathrm{~kg})\end{array}$ & 937040 \\
\hline \multicolumn{3}{|c|}{ The optimal solution for factory $f$} \\
\hline $\begin{array}{c}\text { Process } \\
\text { number } \\
\qquad i\end{array}$ & $\begin{array}{l}\text { Number of machines in process } i \\
\qquad n_{f_{i}}^{m a c h} \text { (units) }\end{array}$ & $\begin{array}{l}\text { Quantity of materials in process } i \\
\qquad q_{f_{i}}^{r}(\mathrm{~kg})\end{array}$ \\
\hline 1 & 4 & 937040 \\
\hline 2 & 40 & 918299 \\
\hline 3 & 3 & 889824 \\
\hline 4 & 5 & 868344 \\
\hline 5 & 13 & 850660 \\
\hline 6 & 13 & 840467 \\
\hline 7 & 60 & 835940 \\
\hline 8 & 4 & 831540 \\
\hline \multicolumn{2}{|c|}{ Number of manufacturing products } & $8,941,290$ sacks \\
\hline
\end{tabular}




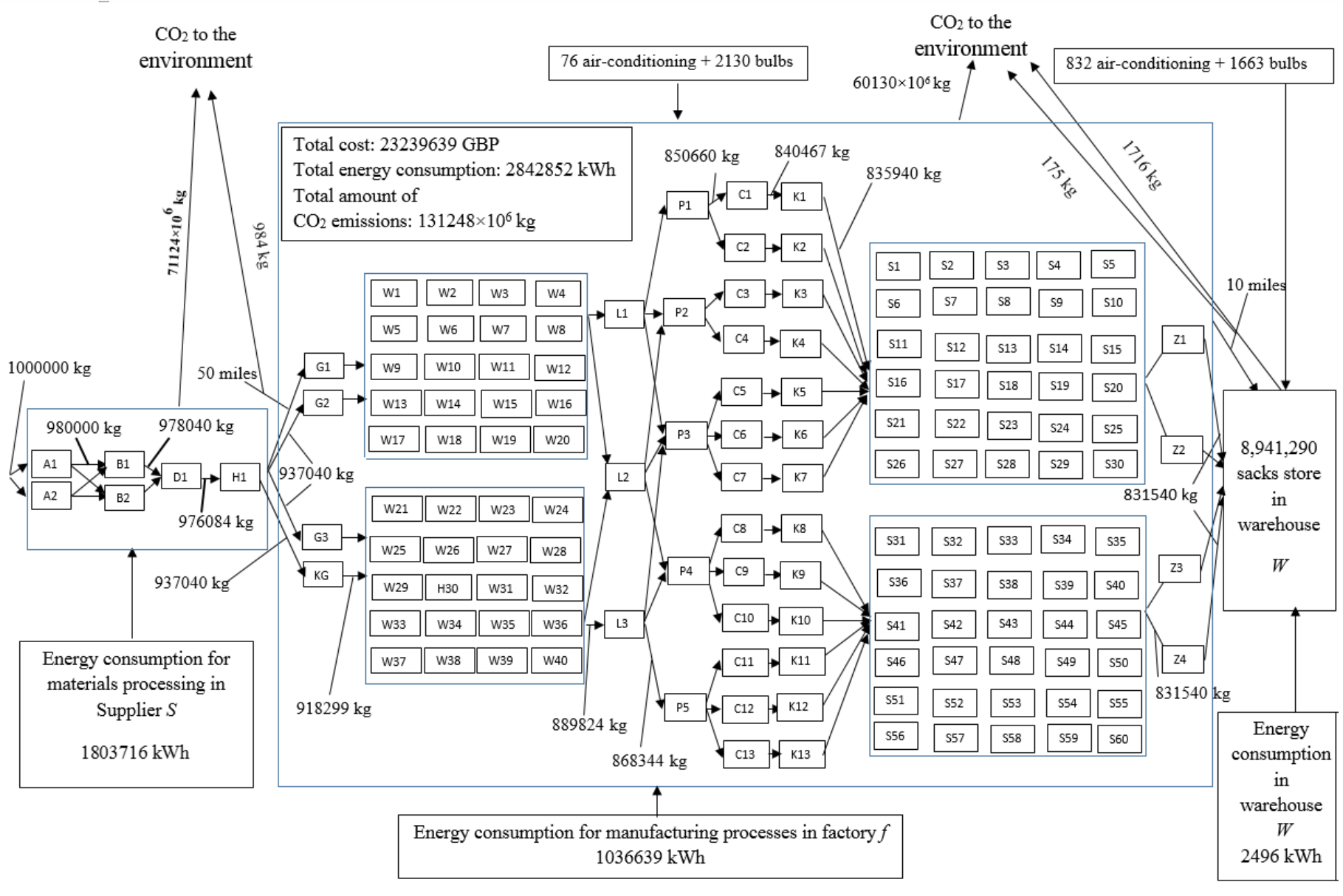

Fig.4. An optimal configuration for the sustainable manufacturing system design 


\section{Conclusion}

Design engineers normally focus on the traditional key-factors e.g. system efficiency and operating capacity when designing a manufacturing system lacking behind the growing interest in environmental aspects. This work solves a sustainable manufacturing design problem via the formulation of a multiple objectives programming model considering economic, energy and environmental aspects. Parameters collected from a plastic and woven sacks manufacturer were used for validating the efficacy of the developed model. The study indicates that the developed multi-objective programming model can be employed as a decision-making tool used for reconfiguring the design of a conventional manufacturing system incorporating economic and ecological aspects. This research also helps decision makers in revealing a compromised solution among conflicting objectives i.e. minimization of total cost, energy consumption and environmental impacts.

Future work should focus on improving the developed model by considering a multi-period multi-objective model and formulating the social impact as an objective function when configuring the SMS. 


\section{References}

Dombrowski, U., Schmidt, S., \& Schmidtchen, K. (2014). Analysis and integration of design for X approaches in lean design as basis for a lifecycle optimized product design. Procedia CIRP, 15, 385-390.

S. Lind, B. Krassi, B. Johansson, J. Viitaniemi, J. Heilala, S. Stahre, S. Vatanen, A. Fasth, and C. Berlin, "SIMTER: A Production Simulation Tool for Joint Assessment of Ergonomics, Level of Automation and Environmental Impacts”, In the 18th FAIM, Stockholm, pp 5-49, 2008.

M. Paju, J. Heilala, M. Hentula, A. Heikkila, B. Johansson, S. Leong, and K. Lyons, "Framework and indicators for a sustainable manufacturing mapping methodology", In the WSC, IEEE, Baltimore, MD, pp 3411-3422, 2010.

H. Taghdisian, M. R. Pishvaie, and F. Farhadi, "Multi-objective optimization approach for green design of methanol plant based on CO2-efficeincy indicator," Journal of Cleaner Production, 2014.

Q. Wang, S. Lassalle, A. R. Mileham, and G. W, Owen, “Analysis of a linear walking worker line using a combination of computer simulation and mathematical modeling approaches," Journal of Manufacturing Systems, vol. 28, 2009 , pp. 64-70.

J. Heilala, S. Vatanen, H. Tonteri, J. Montonen, S. Lind, B. Johansson, and J. Stahre, "Simulation-based sustainable manufacturing system design,” Proceedings of the 2008 Winter Simulation Conference, pp. 1922-1930, 2008.

C. Wang, M. Larsson, C. Ryman, C. E. Grip, J. O. Wikström, A. Johnsson, and J. Engdahl, “A model on CO2 emission reduction in integrated steelmaking by optimization methods," IJER, vol. 32, pp. 1092-1106, 2008.

M. Branham, T. G. Gutowski, A. Jones, and D. P. Sekulic, “A thermodynamic framework for analyzing and improving manufacturing processes," In ISEE. IEEE, San Francisco, CA, 2008, pp. 1-6.

G. Guillén. Gosálbez, and I. E. Grossmann, "Optimal design and planning of sustainable chemical supply chains under uncertainty,” AIChE Journal, vol. 55, No. 1, 2009, pp. 99-121.

V. Sahar, B. Arijit, and P.J. Byrne, “A Case Analysis Of A Sustainable Food Supply Chain Distribution System-A MultiObjective Approach”. International Journal of Production Economics, 152, pp71-87, 2014.

Vahdani, B., Tavakkoli-Moghaddam, R., Modarres, M. and Baboli, A. (2012) "Reliable design of a forward/reverse logistics network under uncertainty: a robust-M/M/c queuing model”, Transport. Res. Part E, VOL. 48 NO. 6, pp. $1152-1168$.

T. Abdallah, A. Diabat, and D.Simchi-Levi, “A carbon sensitive supply chain network problem with green procurement", proceedings of the $40^{\text {th }}$ International Conference in Computers and Industrial Engineering (CIE), pp.1-6, IEEE.2010.

Kannan, G., Sasikumar, P. and Devika, K. (2012), “A genetic algorithm approach for solving a closed loop supply chain model: a case of battery recycling”, Appl. Math.Model, VOL. 34 NO. 3, pp. 655-670.

Zhang, Y., Zhang, G., Du, W., Wang, J., Ali, E., and Sun, S. (2015). An optimization method for shopfloor material handling based on real-time and multi-source manufacturing data. International Journal of Production Economics, 165, $282-292$. 
F. Wang, X. Lai, and N. Shi, “A multi-objective optimization for green supply chain network design, Decision Support Systems, 51, 2011, pp. 262-269.

R. Jamshidi, S. F, Ghomi, and B. Karimi, "Multi-objective green supply chain optimization with a new hybrid memetic algorithm using the Taguchi method,” Scientia Iranica, vol. 19, No. 6, 2012, pp. 1876-1886.

Niknamfar, A.H. (2015), "Multi-objective production-distribution planning based on vendor-managed inventory strategy in a supply chain”, VOL. 115 NO. 6, pp. $1086-1112$.

Bortolini, M., Faccio, M., Ferrari, M., Gamberi, M., and Pilati, F. 2016. Fresh food sustainable distribution: cost, delivery time and carbon footprint three-objective optimization, Journal of Food Engineering, 174, 56-67.

EPA, "The Lean and Environment Toolkit. U.S. Environmental Protection Agency", http://www.epa.gov/lean/toolkit/index.htm accessed June 26, 2008.

R. Nujoom, A. Mohammed, Q. Wang and N. Bennett, "The multi-objective optimization model for a sustainable manufacturing system design. In Renewable Energy Research and Applications (ICRERA), IEEE International Conference on, 2016, pp. 1134-1140.

Chankong, V., \& Haimes, Y. (1983). Multi-objective decision making theory and methodology. New York: Elsevier Science.

Tzeng, G.H., Chiang, C.H., Li, C.W., 2007. Evaluating intertwined effects in e-learning programs: a novel hybrid MCDM model based on factor analysis and DEMATEL. Expert Syst. Appl. 32 (4), 1028e1044.

Charnes, A., Cooper, W.W., Ferguson, R. (1955). Optimal estimation of executive compensation by linear programming. Manag. Sci. 1, 138-151.

Mohammed, A., Wang, Q., Li, X. (2017) "A cost-effective decision-making algorithm for an RFID-enabled HMSC network design: A multi-objective approach", Industrial Management \& Data Systems, 117, 9, 1782-1799 